\title{
Neuronal Activity Regulates Correlated Network Properties of Spontaneous Calcium Transients in Astrocytes In Situ
}

\author{
Fernando Aguado, ${ }^{*}$ Juan F. Espinosa-Parrilla, ${ }^{*}$ María A. Carmona, and Eduardo Soriano \\ Department of Cell Biology and Barcelona Science Park, University of Barcelona, Barcelona E-08028, Spain
}

Spontaneous neuronal activity is essential to neural development. Until recently, neurons were believed to be the only excitable cells to display spontaneous activity. However, cultured astrocytes and, more recently, astrocytes in situ are now known to exhibit spontaneous $\mathrm{Ca}^{2+}$ transients. Here we used $\mathrm{Ca}^{2+}$ imaging of astrocytes from transgenic mice for the simultaneous monitoring of $\left[\mathrm{Ca}^{2+}\right]_{i}$ changes in large numbers of astrocytes. We found that spontaneous activity is a common property of most brain astrocytes that is lost in response to a lesion. These spontaneous $\left[\mathrm{Ca}^{2+}\right]_{\mathrm{i}}$ oscillations require extracellular and intracellular $\mathrm{Ca}^{2+}$. Moreover, network analysis revealed that most astrocytes formed correlated networks of dozens of these cells, which were synchronous with both astrocytes and neurons. We found that decreasing spontaneous $\left[\mathrm{Ca}^{2+}\right]_{\mathrm{i}}$ transients in neurons by TTX does not alter the number of active astrocytes, although it impairs their synchronous network activity. Conversely, bicuculline-induced epileptic patterns of $\left[\mathrm{Ca}^{2+}\right]_{i}$ transients in neurons cause an increase in the number of active astrocytes and in their network synchrony. Furthermore, activation of non-NMDA and NMDA ionotropic glutamate receptors is required to correlate astrocytic networks. These results show that spontaneous activity in astrocytes and neurons is patterned into correlated neuronal/astrocytic networks in which neuronal activity regulates the network properties of astrocytes. This network activity may be essential for neural development and synaptic plasticity.

Key words: astrocyte; calcium; epilepsy; correlated networks; GFAP/GFP mice; glutamate receptors; injury; spontaneous activity; synchrony
Spontaneous neuronal activity is an important property of the developing brain. It is essential for neuronal migration, axonal and dendritic growth, and the formation and refinement of neural connections and synapses (Katz and Shatz, 1996; Komuro and Rakic, 1998; Feller, 1999; Spitzer et al., 2000; Stellwagen and Shatz, 2002). Changes and oscillations in intracellular $\mathrm{Ca}^{2+}$ concentrations $\left(\left[\mathrm{Ca}^{2+}\right]_{i}\right)$ are the main mechanism by which spontaneous activity controls neural development (Berridge, 1998). An additional feature of this activity is the occurrence of synchronous patterns of coactive neurons, which amplify activity functions in neural development by coordinating neural activity and gene expression in vast numbers of cells (Yuste et al., 1992; Buonanno and Fields, 1999; Feller, 1999; O’Donnovan, 1999; Wong, 1999; Ben-Ari, 2001).

Until recently, neurons were considered to be the only excitable cells to participate in spontaneous and evoked neurotransmission and in the synaptic control of brain excitability. However, cultured and in situ astroglial cells respond to various stimuli such as neurotransmitters, hormones, and mechanical stress by increasing $\left[\mathrm{Ca}^{2+}\right]_{\mathrm{i}}$ (Verkhratsky et al., 1998). For instance, neuronal stimulation triggers glutamate- and GABA-mediated $\left[\mathrm{Ca}^{2+}\right]_{\mathrm{i}}$ in-

Received Oct. 24, 2001; revised July 31, 2002; accepted Aug. 6, 2002.

This work was supported by grants from Comisión Interministerial de Ciencia y Tecnología, Fondo de Investigación Sanitaria, and Fondo Europeo de Desarróllo Regional (FIS01-1684 and SAF01-3098 to F.A., and FEDER 2FD97-1760-C03-01, SAF98-0106, and SAF01-134 to E.S.). M.A.C. and J.F.E.-P. are recipients of fellowships from the Ministerio de Educación y Ciencia. We thank Dr. Rafael Yuste (Columbia University, New York, NY) for kindly providing the methodology used and for valuable suggestions, Dr. Alfonso Araque (Cajal Institute, Madrid, Spain) for helpful comments, and Robin Rycroft for editorial assistance.

${ }^{*}$ F.A. and J.F.E.-P. contributed equally to this work.

Correspondence should be addressed to Dr. Fernando Aguado, Barcelona Science Park, University of Barcelona, Modular Building (A1-S1), Josep Samitier, 1-5, Barcelona E-08028, Spain. E-mail: aguado@medicina.ub.es.

Copyright (C) 2002 Society for Neuroscience $0270-6474 / 02 / 229430-15 \$ 15.00 / 0$ creases in astrocytes in brain slices (Dani et al., 1992; Porter and McCarthy, 1996; Pasti et al., 1997; Kang et al., 1998); conversely, astrocyte $\left[\mathrm{Ca}^{2+}\right]_{\mathrm{i}}$ increases modulate EPSCs and IPSCs in neurons through glial-released glutamate (Pasti et al., 1997, 2001; Araque et al., 1998a,b; Bezzi et al., 1998; Parpura and Haydon, 2000). Thus, there may be reciprocal signaling between astrocytes and neurons, thereby dynamically regulating nerve excitability (Cooper, 1995; Vesce et al., 1999; Carmignoto, 2000; Araque et al., 2001; Bezzi and Volterra, 2001; Haydon, 2001).

Cortical astroglial cells in vitro display spontaneous $\left[\mathrm{Ca}^{2+}\right]_{\mathrm{i}}$ oscillations (Fatatis and Rusell, 1992; Charles, 1994; HarrisWhite et al., 1998). Recently, it has been shown that astrocytes from brain slices also show spontaneous $\left[\mathrm{Ca}^{2+}\right]_{\mathrm{i}}$ oscillations in situ (Parri et al., 2001; Nett et al., 2002). Moreover, spontaneous astrocytic oscillations in the thalamus trigger neuronal excitation through the NMDA glutamate receptor (Parri et al., 2001). By imaging large numbers of astrocytes in transgenic mice targeted to identify these glial cells, here we show that spontaneous $\left[\mathrm{Ca}^{2+}\right]_{\mathrm{i}}$ transients are a common feature of resting astrocytes in all the brain regions examined, but they are lost in reactive astrocytes. Most of these spontaneously active astrocytes were synchronous, forming complex correlated networks of up to dozens of astrocytes, which in turn were synchronous with spontaneous neuronal networks. Most importantly, by blocking action potentials with tetrodotoxin (TTX) or by regulating neuronal activity with $\mathrm{GABA}_{\mathrm{A}}$ and ionotropic glutamate receptor antagonists, we show that spontaneous neuronal activity controls the properties and complexity of astrocytic network activity.

\section{MATERIALS AND METHODS}

Animals. Glial fibrillary acidic protein (GFAP)/green fluorescent protein (GFP) transgenic mice (Zhuo et al., 1997) were purchased from Jackson Laboratories (Bar Harbor, ME). The GFAP/GFP colony was kept under 

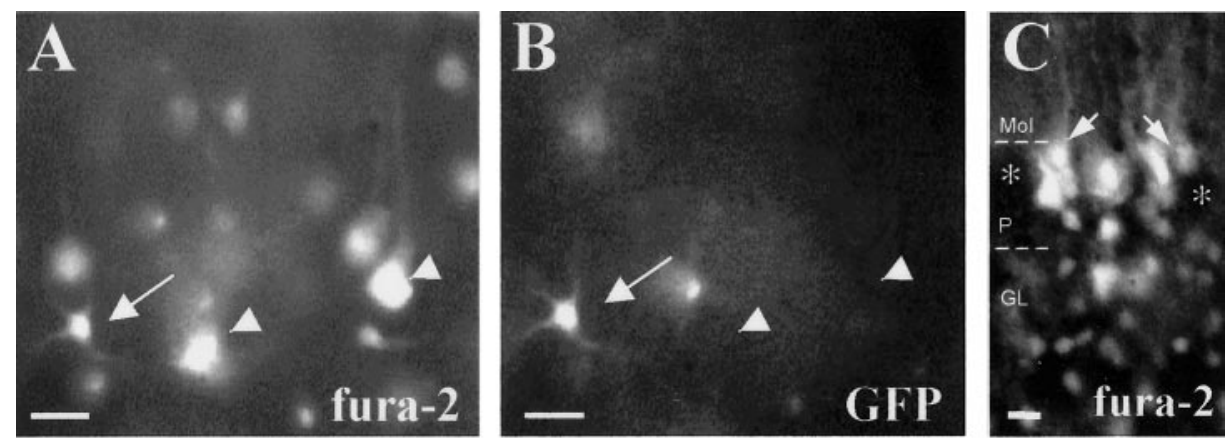

D neocortex (P7)
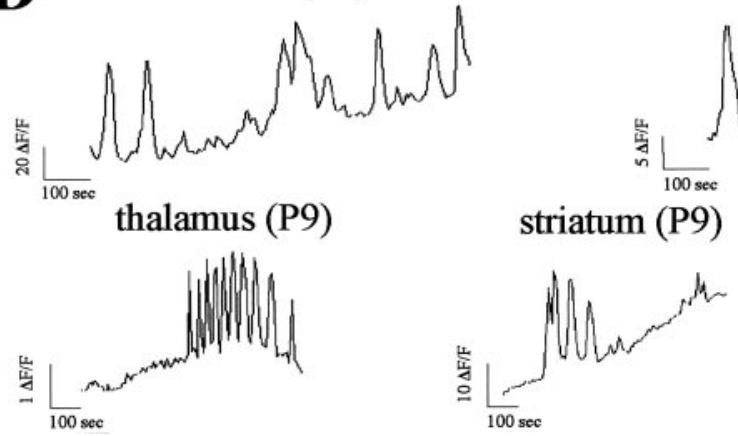

hippocampus (P6)

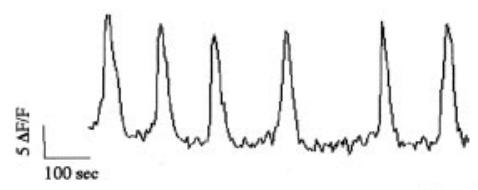

Bergmann glia (P25)

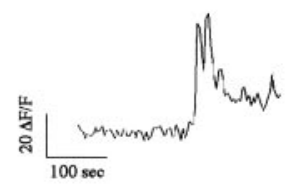

Figure 1. Spontaneous $\left[\mathrm{Ca}^{2+}\right]_{i}$ oscillations are a common feature of astrocytes in situ. $A, B$, Paired fluorescence photomicrographs showing the same field under fura-2 $(A)$ and GFP $(B)$ fluorescence, illustrating GFP/fura-2labeled astrocytes in layers II-III of the neocortex of P7 GFAP/GFP mice. Double-labeled astrocytes are indicated by arrows; pyramidal neurons lacking GFP fluorescence are indicated by arrowheads. $C$, Fluorescence photomicrograph illustrating fura-2-loaded Bergmann glial cells (arrows) in a P25 cerebellar slice. Purkinje cells, devoid of fura-2 loading, are labeled by asterisks. $D$, Representative spontaneous changes of fura-2 fluorescent signal $(\Delta F / F)$ over time recorded in astrocyte somata located in distinct CNS regions of P6-P25 GFAP/GFP mice. The spontaneous activity profile of the neocortical astrocyte corresponds to the astrocyte shown in $A$ and $B$. Note bursting and oscillatory $\mathrm{Ca}^{2+}$ changes in the examples shown in the thalamus and hippocampus, respectively. Scale bars: $A, B, 20 \mu \mathrm{m} ; C, 12 \mu \mathrm{m}$. $\mathrm{Mol}$, Molecular layer; $P$, Purkinje cell layer; $G L$, granule cell layer. controlled temperature $\left(22 \pm 2^{\circ} \mathrm{C}\right)$, humidity (40-60\%), and light $(12 \mathrm{hr}$ cycles) and treated in accordance with the European Community Council Directive (86/609/EEC). Stab wound lesions were made as described previously (Mathewson and Berry, 1985). In brief, postnatal day (P) 22 GFAP/ GFP mice were anesthetized with ketamine-xylazine injections (150 and 6 $\mu \mathrm{g} / \mathrm{g}$, respectively), and their heads were fixed to a stereotactic frame. The cerebral cortex was then stabbed parasagittally with a scalpel blade. The wounds were $1 \mathrm{~mm}$ deep and $3 \mathrm{~mm}$ long and ran $2 \mathrm{~mm}$ away from the midline over the right parietal cortex. Lesioned mice were used $2 \mathrm{~d}$ later. Reactive astrocytes were recognized by the increased GFP fluorescence signal and their typical hypertrophied morphology (Latov et al., 1979; Mathewson and Berry, 1985; Salhia et al., 2000; Nolte et al., 2001).

Materials. Fura-2 AM and pluronic acid were purchased from Molecular Probes (Eugene, OR). ( \pm )-2-amino-5-phosphonopentanoic acid (APV), 6-cyano-7-nitroquinoxaline-2, 3-dione (CNQX), (+)- $\alpha$-methyl-4carboxyphenylglycine (MCPG), (-)-bicuculline methiodide (BMI), TTX, ( \pm )-2-octanol, 1-heptanol, $18 \alpha$-glycyrrhetinic acid $(\alpha$-GA), trans(1S,3R)-1-amino-1,3-cyclopentanedicarboxylic acid ( $t$-ACPD), and EGTA were obtained from Sigma (St. Louis, MO). Suramin, 4-chloro$\mathrm{m}$-cresol (4-CmC), and thapsigargin were from CalbiochemNovabiochem Corporation (La Jolla, CA), and CGP55845 was from Tocris Cookson (Buckhurst Hill, UK). Drugs were dissolved in distilled water and DMSO.

$\mathrm{Ca}^{2+}$ imaging. P2-P24 and adult (3-11 month old) GFAP/GFP mice were anesthetized by hypothermia or ketamine-xylazine injections $(150$ and $6 \mu \mathrm{g} / \mathrm{g}$, respectively). Their brains and spinal cords were removed and placed in cold artificial CSF (ACSF) containing (in mM): $\mathrm{NaCl} 120$, $\mathrm{KCl}$ 3, D-glucose 10, $\mathrm{NaHCO}_{3} 26, \mathrm{NaH}_{2} \mathrm{PO}_{4} 2.25, \mathrm{CaCl}_{2} 2, \mathrm{MgSO}_{4} 1, \mathrm{pH}$ 7.4, bubbled with $95 \% \mathrm{O}_{2}$ and $5 \% \mathrm{CO}_{2}$. Coronal and horizontal tissue slices (300 $\mu \mathrm{m}$ thick) were cut with a vibratome and before imaging were kept for at least $1 \mathrm{hr}$ in a storage chamber containing ACSF bubbled continuously with $95 \% \mathrm{O}_{2}$ and $5 \% \mathrm{CO}_{2}$ at room temperature $\left(22-25^{\circ} \mathrm{C}\right)$. $\left[\mathrm{Ca}^{2+}\right]_{\mathrm{i}}$ in slices was measured with the membrane-permeant acetoxymethyl ester of fura-2 AM dissolved in DMSO with $0.001 \%$ pluronic acid. The tissue slices were incubated in 3-5 $\mu \mathrm{l}$ of $5 \mathrm{~mm}$ fura- $2 \mathrm{AM}$ for $2 \mathrm{~min}$ and then in $3 \mathrm{ml}$ of $10 \mu \mathrm{M}$ dye in ACSF for $30 \mathrm{~min}$, as described previously (Schwartz et al., 1998; Aguiló et al., 1999). Slices were always maintained in oxygenated ACSF.

Fura-2-loaded slices were transferred to a continuously superfused recording chamber on the stage of a fluorescent upright microscope (BX50WI; Olympus, Tokyo, Japan) equipped with 380 and $340 \mathrm{~nm}$ excitation filters and differential interference contrast optics. Recordings of $\left[\mathrm{Ca}^{2+}\right]_{\mathrm{i}}$ changes were imaged with $20 \times$ and $40 \times$ water-immersion objectives at room temperature $\left(22-25^{\circ} \mathrm{C}\right)$. Images were captured with a silicon-intensifier tube camera (Hamamatsu C2400-08) and a frame grabber (LG-3; Scion Corporation, Frederick, MD) connected to a Macintosh computer (Apple Computers, Cupertino, CA). Fura-2 fluorescence images were collected at $4 \mathrm{sec}$ intervals ( 15 frames were averaged for each time point) at a single excitation wavelength using the 380 $\mathrm{nm}$ bandpass filter over periods of up to 20 min controlled by the NIH Image program. To prevent photobleaching, a shutter (UniBlitz) controlled by custom-written macros was used.

Network analysis. To analyze coactive networks of optical recordings, we followed a methodology described previously in detail (Schwartz et al., 1998; Aguiló et al., 1999). Changes in the fluorescence in multiple cells were analyzed with a program written in Interactive Data Language (Research Systems, Inc., Boulder, CO). The fluorescence change over time was defined as $\Delta F / F=\left(F_{0}-F_{1}\right) /\left(F_{0}\right)$. The onset of each calcium transient for every cell was determined using an algorithm that defined the onset as the frame after which the $\Delta F / F$ change was larger than a given set threshold, typically a change of three to five pixel value units per frame. This threshold algorithm was very sensitive but produced false positives in some cells in which noise baseline values were scored as transients, as determined by visual inspection. The $\left[\mathrm{Ca}^{2+}\right]_{\mathrm{i}}$ transients detected by the program were inspected carefully, and spurious events were canceled. The time of initiation of each calcium transient for each cell was marked in a raster plot. These plots were used to calculate the matrix of asymmetric correlation coefficients between all cell pairs, i.e., the proportion of times that a cell becomes active when another cell is also active. Contingency tables were then used, and $\chi^{2}$ tests were run to detect which of the correlation coefficients was significantly greater than expected. Significant correlation coefficients were used to generate a correlation map on which lines link neurons whose asymmetric correlation coefficients are significant $(p<0.01)$ and on which the thickness of a line connecting any two cells represents the size of the greater asymmetric correlation coefficient between the cells.

To test whether the $\left[\mathrm{Ca}^{2+}\right]_{\mathrm{i}}$ transients showed associations between the cells within a network, we measured the number of simultaneous activations in a recording and used it as a statistical test. To determine its $p$ value, the distribution of the statistics under the null hypothesis of independent transients was computed by Monte Carlo simulation. The $p$ value was then calculated as the proportion of the 1000 replications in which the statistical test exceeded the statistical test computed from the real data. To simulate independent realizations of the transients, the number of transients in each train was preserved, but the times were chosen at random. This approach is equivalent to assuming that the 

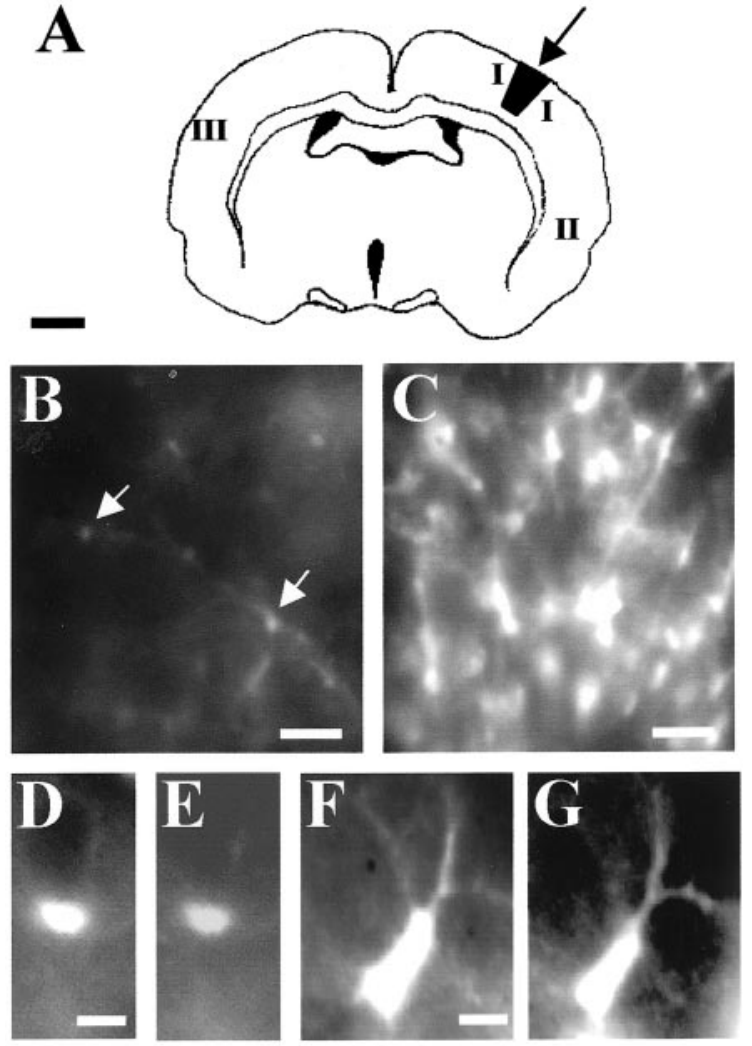

H

resting astrocyte

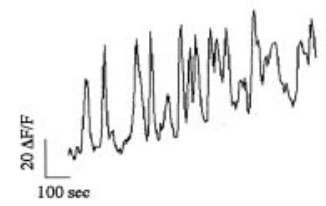

reactive astrocyte

Figure 2. Reactive astrocytes lack spontaneous $\left[\mathrm{Ca}^{2+}\right]_{\mathrm{i}}$ transients. $A$, Schematic diagram illustrating the location of the stab wound lesion in the parietal cortex (arrow) of P24 GFAP/GFP neocortex and the areas in which spontaneous astrocytic activity was recorded $48 \mathrm{hr}$ after the lesion $(I, I I$, and $I I I) . B, C$, Low-magnification photomicrographs showing GFAP/GFP-positive astrocytes (arrows) in areas III ( $B$, resting astrocytes) and I ( $C$, reactive astrocytes). Note that the number of astrocytes and the intensity of GFP fluorescence are markedly increased in astrocytes around the lesion. $D-G$, Higher-magnification photomicrographs of typical GFAP/GFP-positive resting astrocytes in area III $(D, E)$ and reactive GFAP/GFP-positive astrocytes in area I $(F, G)$. Intense GFP positive-reactive astrocytes have larger somata and hypertrophied processes $(F)$ than resting GFP cells $(D)$. In both resting $(E)$ and reactive $(G)$ astrocytes, the fura- 2 indicator was loaded similarly. $H,\left[\mathrm{Ca}^{2+}\right]_{\mathrm{i}}$ profiles over $800 \mathrm{sec}$ of representative GFAP/GFP-positive astrocytes recorded near the lesion site (reactive astrocyte; area I) and in the contralateral hemisphere (resting astrocyte; area III). Scale bars: $A, 1 \mathrm{~mm} ; B, C, 40 \mu \mathrm{m}$; $D-G, 5 \mu \mathrm{m}$.

distributions of the transients behave as Poisson processes with varying underlying rates. We tested this assumption with a control computation of $p$ values using randomized starting times for each train and wrapping around the ends and found no different results. We also performed an additional test on each sample to detect groups of cells that were activated simultaneously more than once, and we used this number as the statistical test (Schwartz et al., 1998; Aguiló et al., 1999). Monte Carlo simulations were again used to estimate the significance of this statistical test.

The Student's $t$ test was used to compare all measurements. Data are expressed as mean \pm SEM.

\section{RESULTS}

\section{Resting, but not reactive astrocytes, display spontaneous $\left[\mathrm{Ca}^{2+}\right]_{\mathrm{i}}$ transients in the CNS}

To examine whether astrocytes have spontaneous $\left[\mathrm{Ca}^{2+}\right]_{\mathrm{i}}$ oscillations in situ, we used acute brain slices from transgenic mice that express GFP under the control of the GFAP promoter (Zhuo et al., 1997). GFAP/GFP-positive cells displayed the typical size and morphology of astrocytes in all brain regions and ages examined (Figs. $1 A-C, 2 B, D, 4 A, B$ ), which was consistent with earlier studies (Zhuo et al., 1997; Nolte et al., 2001). When transgenic slices were loaded with the $\mathrm{Ca}^{2+}$ indicator fura-2, neurons labeled with this $\mathrm{Ca}^{2+}$ indicator did not express GFP (Fig. 1A,B). Thus, fura-2 loading of GFAP/GFP transgenic slices allowed the unequivocal identification of large numbers of astrocytes.

We monitored spontaneous $\left[\mathrm{Ca}^{2+}\right]_{\mathrm{i}}$ changes with a highresolution imaging system described elsewhere (Schwartz et al., 1998; Aguiló et al., 1999). Time-lapsed recordings revealed spontaneous $\left[\mathrm{Ca}^{2+}\right]_{\mathrm{i}}$ changes in numerous GFAP/GFP-positive cell bodies corresponding to astrocytes. These spontaneous astrocytic $\left[\mathrm{Ca}^{2+}\right]_{\mathrm{i}}$ changes occurred in various $\mathrm{CNS}$ regions, including the neocortex, hippocampus, entorhinal cortex, striatum, cerebellum, thalamus, hypothalamus, and spinal cord (Fig. $1 A-D$ ). Astrocytic $\left[\mathrm{Ca}^{2+}\right]_{\mathrm{i}}$ transients were detected as early as P2 (the earliest stage examined) and were common during the first 2 postnatal weeks. For instance, $71 \%$ of GFAP/GFP-positive astrocytes recorded at P5-P9 (167 of 236 cells in 11 slices) showed spontaneous $\mathrm{Ca}^{2+}$ signaling in the hippocampus (Figs. $1 D, 4 C, D$ ). Because the number of fura-2-loaded cells decreases with age (Yuste and Katz, 1991), fewer GFAP/GFP-positive astrocytes were loaded with fura-2 after P15. However, almost half of these fura-2loaded astrocytes exhibited spontaneous $\left[\mathrm{Ca}^{2+}\right]_{\mathrm{i}}$ transients at P24 (e.g., 44\%; 24 of 55 cells in the neocortex) (Fig. 2F). Spontaneous $\left[\mathrm{Ca}^{2+}\right]_{\mathrm{i}}$ oscillations were still detected in adult astrocytes, although less frequently (e.g., 26\%; 8 of 31 cells in four neocortical slices from two animals) than in postnatal stages. Most of these spontaneous $\left[\mathrm{Ca}^{2+}\right]_{\mathrm{i}}$ events were long (typically, 10-45 sec) (Figs. $1 D, 2 F, 3 B-D, 4 C$ ) and variable in amplitude, even in the same brain region. The frequency and patterns of activity were variable and included random profiles, but also rhythmic oscillations and bursting activity (Fig. 1D). We conclude that spontaneous $\left[\mathrm{Ca}^{2+}\right]_{\mathrm{i}}$ transients are a common feature of developing and adult astrocytes in the CNS.

Astrocytes have a number of functions, including homeostatic control of the extracellular "milieu," the regulation of synaptic function, and a protective role after CNS injury (Kettenmann and Ransom, 1995). To study whether spontaneous $\left[\mathrm{Ca}^{2+}\right]_{\mathrm{i}}$ changes in astrocytes depend on their functional state, we determined whether these spontaneous oscillations persisted in reactive astrocytes. We compared $\mathrm{Ca}^{2+}$ signaling in reactive and resting astrocytes $48 \mathrm{hr}$ after stab wound injuries (Mathewson and Berry, 1985) in the neocortex of P22 GFAP/GFP transgenic mice (Fig. 2A). As described for endogenous GFAP and for transgenic reporter expressions (Latov et al., 1979; Mathewson and Berry, 1985; Salhia et al., 2000; Nolte et al., 2001), large numbers of astrocytes located around the wound lesion (100-500 $\mu \mathrm{m})$ exhibited strong GFP expression in GFAP/GFP transgenic slices, in both the cell bodies and processes (Fig. $2 B, C$ ). Moreover, astrocytes in the hemisphere ipsilateral to the wound showed the typical hypertrophy described for stab injury-induced reactive astrogliosis (Latov et al., 1979; Mathewson and Berry, 1985; 


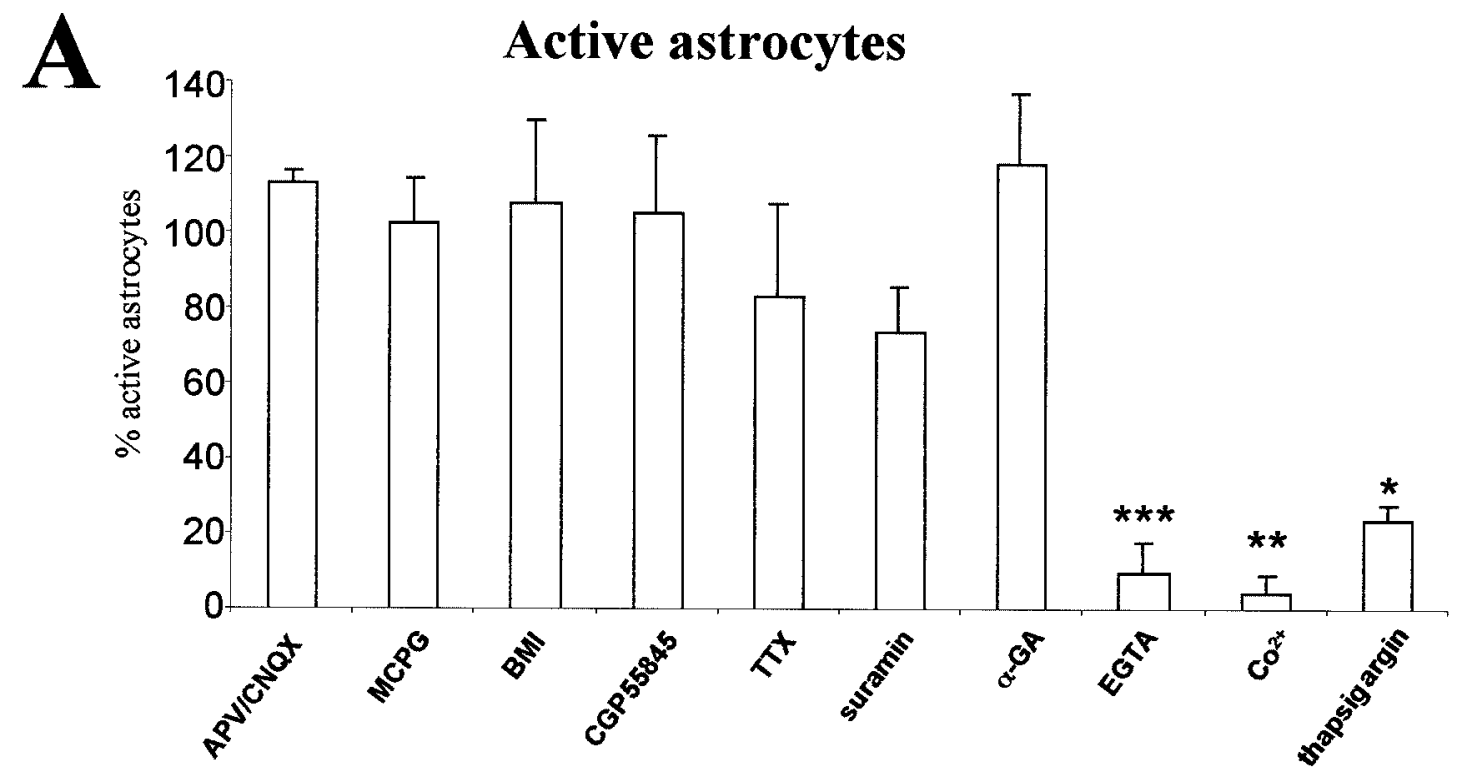

B
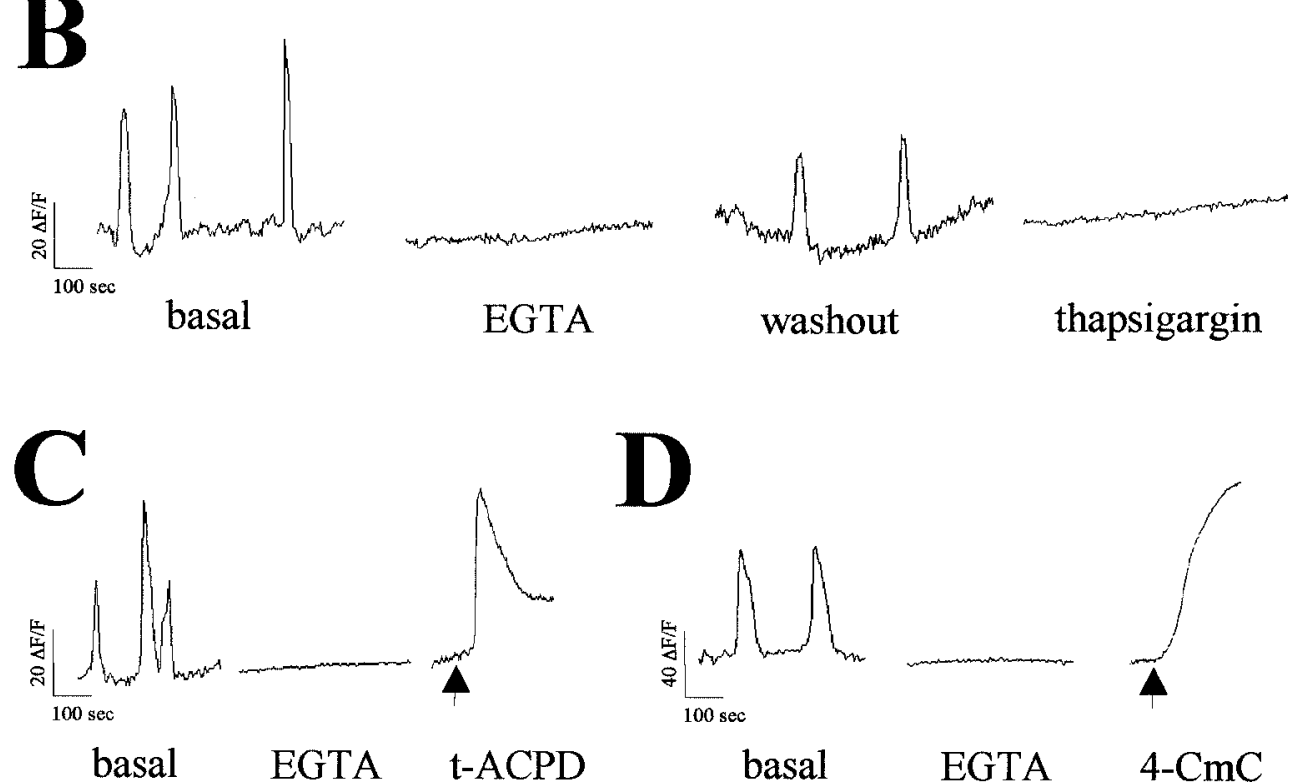

D

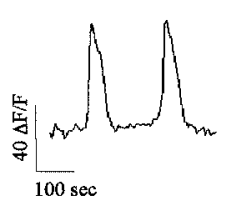

basal

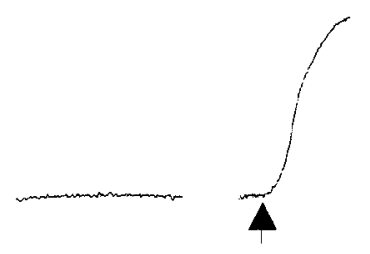

EGTA 4-CmC

Figure 3. Pharmacological analysis of spontaneous $\left[\mathrm{Ca}^{2+}\right]_{\mathrm{i}}$ transients in hippocampal astrocytes. $A$, Histograms illustrating the percentage of hippocampal P2-P6 GFAP/GFP-positive astrocytes showing spontaneous $\mathrm{Ca}^{2+}$ activity after administration of a range of neurotransmitter receptor antagonists, TTX, and blockers of $\mathrm{Ca}^{2+}$ mobilization. Significant reductions are observed after EGTA, $\mathrm{Co}^{2+}$, and thapsigargin treatments $\left({ }^{*} p<0.05\right.$; $*^{*} p<0.01$; *** $\left.p<0.001\right)$. Each experimental condition was performed in at least three slices. Data are expressed as percentages of control values (mean \pm SEM). B, Representative plots of $\left[\mathrm{Ca}^{2+}\right]_{\mathrm{i}}$ oscillations recorded in the same GFAP/GFP-positive astrocyte from a P6 mouse perfused with normal ACSF (basal and washout), $\mathrm{Ca}^{2+}$-free ACSF (2 mM EGTA, $\left.0 \mathrm{~mm}\left[\mathrm{Ca}^{2+}\right]_{\mathrm{o}}\right)$, and thapsigargin $(2 \mu \mathrm{M}) . C, D,\left[\mathrm{Ca}^{2+}\right]_{\mathrm{i}}$ oscillations recorded in P5 GFAP/GFP-positive astrocytes are abolished after perfusion of $\mathrm{Ca}^{2+}$-free ACSF. Addition of $t$-ACPD and 4-CmC (arrows) to nominally Ca ${ }^{2+}$-free ACSF caused a transient and a progressive increase in $\left[\mathrm{Ca}^{2+}\right]_{\mathrm{i}}$, respectively. Abbreviations are defined in Materials and Methods.

Salhia et al., 2000; Nolte et al., 2001). Thus, although resting astrocytes in the contralateral cortex were $\sim 4 \mu \mathrm{m}$ in size and showed extremely thin processes (Fig. $2 D, E$ ), reactive astrocytes showed increased GFAP/GFP fluorescence and had larger somata ( $\sim 10 \mu \mathrm{m}$ in size) and hypertrophied processes (Fig. $2 F, G)$. Only 2 of 82 reactive astrocytes (imaged at $100-200 \mu \mathrm{m}$ from the lesion) had spontaneous $\left[\mathrm{Ca}^{2+}\right]_{i}$ oscillations, and these were of very low amplitudes (one of seven slices from five animals). Those remaining reactive astrocytes showed no spontaneous $\left[\mathrm{Ca}^{2+}\right]_{\mathrm{i}}$ changes even after $15 \mathrm{~min}$ of recording (Fig. $2 \mathrm{H}$ ). Characteristically, other cell bodies located near the wound that were GFAP/
GFP negative and had the shape and size of neurons showed frequent spontaneous $\left[\mathrm{Ca}^{2+}\right]_{\mathrm{i}}$ transients (data not shown). In contrast, the proportion of GFAP/GFP-positive cells exhibiting these transients increased in proportion to their distance from the lesion (Fig. 2A). For instance, 51\% (19 of 33) and 44\% (24 of 55) of astrocytes exhibited spontaneous $\left[\mathrm{Ca}^{2+}\right]_{i}$ oscillations at $2.5-3$ $\mathrm{mm}$ ipsilateral to the lesion and in the contralateral hemisphere, respectively (six of six slices from four animals) (Fig. 2H). These data indicate that spontaneous $\left[\mathrm{Ca}^{2+}\right]_{\mathrm{i}}$ oscillations are a common functional property of resting astrocytes that is lost when astrocytes respond to a mechanical lesion. 

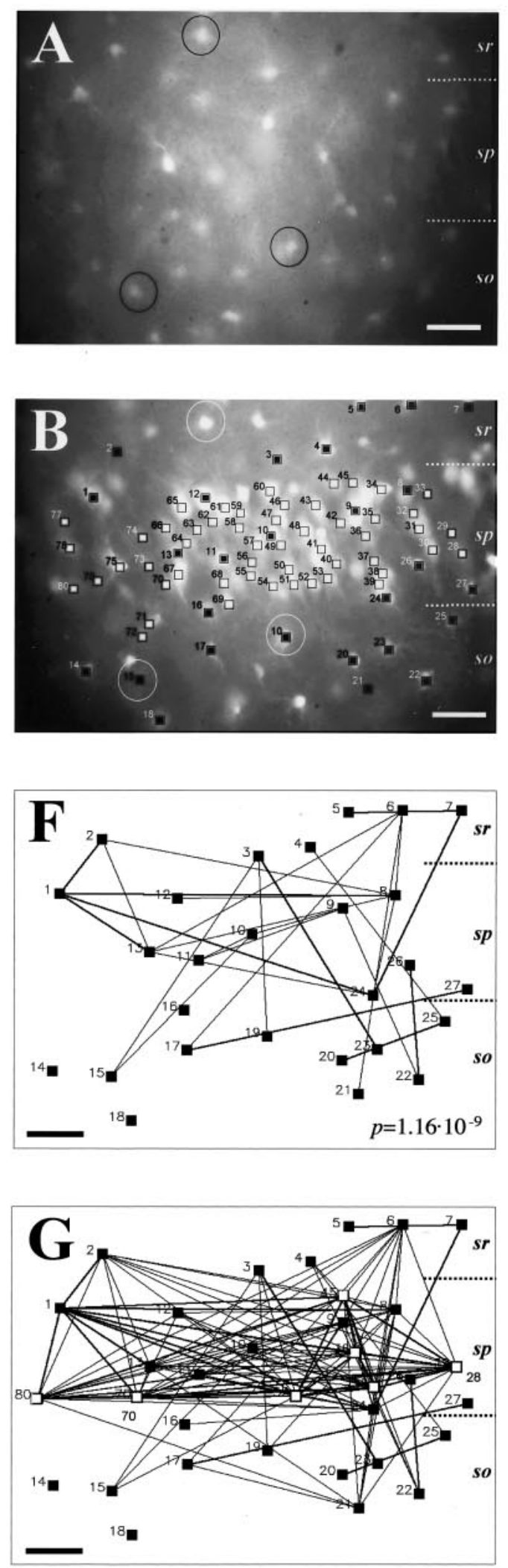
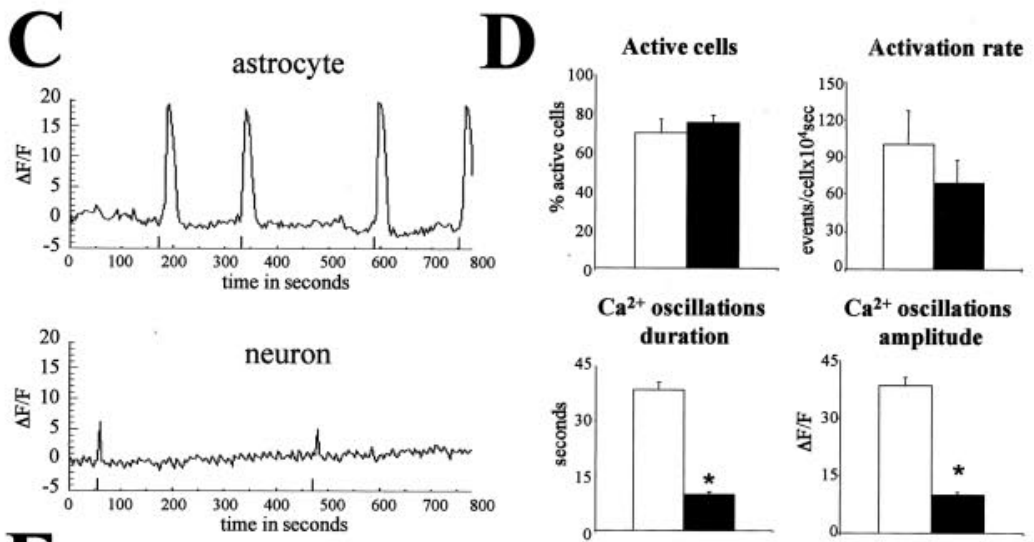

$\mathbf{E}$
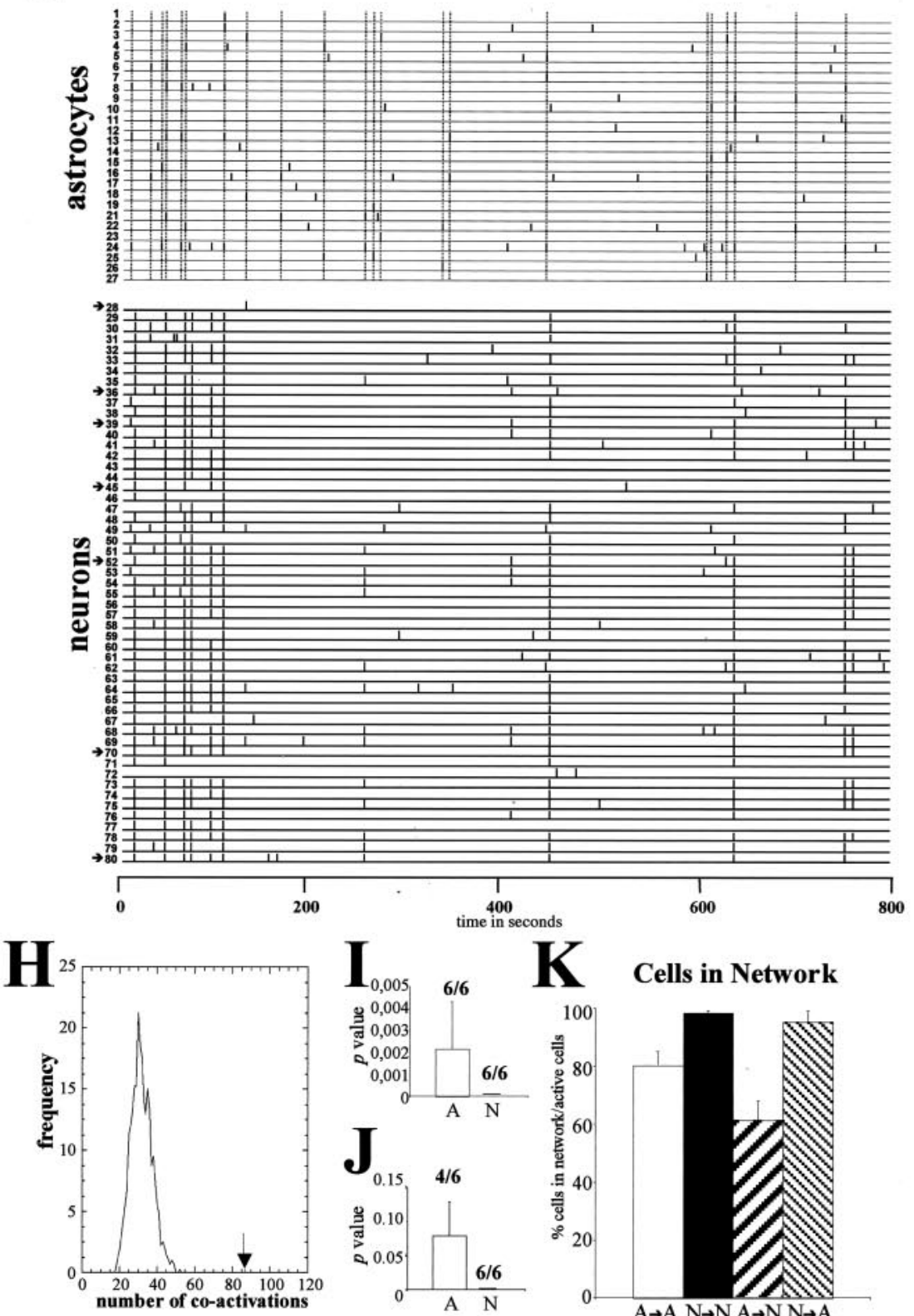

K Cells in Network

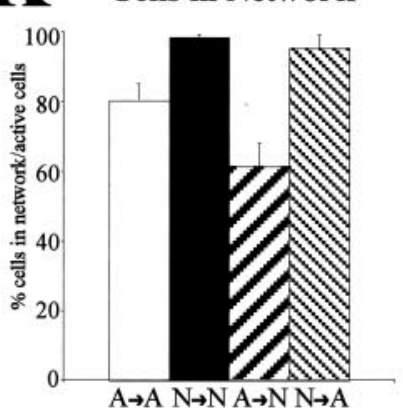

Figure 4. Spontaneous $\left[\mathrm{Ca}^{2+}\right]_{\mathrm{i}}$ oscillations in hippocampal astrocytes belong to correlated neuronal/astrocytic networks. $A, B$, Paired fluorescence photomicrographs illustrating many GFAP/GFP-positive astrocytes $(A)$ in the CA1 region of the hippocampus (P6) that are also labeled with fura-2 (e.g., cells in circles) (B). Fura-2-loaded cells showing spontaneous fluorescence changes over time are labeled by squares. Black (Figure legend continues.) 


\section{Extracellular and intracellular $\mathrm{Ca}^{2+}$ mobilization is required for the generation of spontaneous oscillations in hippocampal astrocytes}

Next, we studied whether activation of neurotransmitter receptors drives spontaneous $\left[\mathrm{Ca}^{2+}\right]_{\mathrm{i}}$ oscillations in GFAP/GFPpositive astrocytes from $\mathrm{P} 2-\mathrm{P} 6$ hippocampal slices. Incubation with antagonists for ionotropic (APV/CNQX, 50 and $20 \mu \mathrm{M}$ ) and metabotropic (MCPG, $1 \mathrm{~mm}$ ) glutamate receptors did not alter the proportion of hippocampal astrocytes displaying spontaneous $\left[\mathrm{Ca}^{2+}\right]_{\mathrm{i}}$ transients (Fig. $3 A$ ). Moreover, no significant changes were observed after addition of antagonists for ionotropic $\mathrm{GABA}_{\mathrm{A}}(\mathrm{BMI}, 30 \mu \mathrm{M})$ and metabotropic GABA B $_{\mathrm{B}}$ (CGP55845, 6 $\mu \mathrm{M})$ GABA receptors (Fig. $3 A$ ). Similarly, incubation with the $\mathrm{Na}^{+}$channel blocker TTX $(2 \mu \mathrm{M})$ did not significantly modify spontaneous activity in astrocytes (Fig. $3 A$ ). In marked contrast to these observations in astrocytes, we found that TTX and the synaptic blockers altered spontaneous $\left[\mathrm{Ca}^{2+}\right]_{\mathrm{i}}$ transients in neurons (see below). These data indicate that the generation of spontaneous astrocytic activity depends neither on the activation of the main neurotransmitter systems nor on action potentials.

Because ATP signaling is a relevant extracellular messenger that activates astrocytes (Fields and Stevens, 2000), we evaluated the participation of purinergic receptors in the generation of spontaneous $\left[\mathrm{Ca}^{2+}\right]_{\mathrm{i}}$ oscillations by blocking $\mathrm{P} 2$ receptors with the antagonist suramin $(100 \mu \mathrm{M})$ in the presence of TTX $(2 \mu \mathrm{M})$ to block suramin effects on neurons (Cunha and Ribeiro, 2000). Again, the proportion of spontaneously active astrocytes was not altered significantly under these conditions (Fig. $3 A$ ). Finally, we studied the requirement of gap junctions in the generation of spontaneous astrocytic activity (Giaume and McCarthy, 1996; Giaume and Venance, 1998). After bath application of $100 \mu \mathrm{M}$ $\alpha$-GA, a gap junction blocker (Davidson and Baumgarten, 1998; Venance et al., 1998), the percentage of spontaneously active astrocytes was not altered (Fig. $3 A$ ). Moreover, perfusion of the slices with the long-chain alcohols octanol and heptanol (1 mM), which uncouple astrocyte gap junctions (Venance et al., 1998), showed similar results (data not shown). These data indicate that neither P2 receptor activation nor gap junctions are essential for the generation of spontaneous astrocytic activity.

To investigate the cellular mechanisms that underlie spontaneous $\left[\mathrm{Ca}^{2+}\right]_{\mathrm{i}}$ transients, we incubated hippocampal slices (P5-P9) with drugs influencing $\mathrm{Ca}^{2+}$ mobilization. Rapid removal of extracellular $\mathrm{Ca}^{2+}$ by perfusion with $\mathrm{Ca}^{2+}$-free ACSF containing 2 mM EGTA blocked spontaneous $\left[\mathrm{Ca}^{2+}\right]_{\mathrm{i}}$ oscillations in 103 of 113 imaged astrocytes (four of six slices from six animals) (Fig. $3 A, B)$. This blocking effect was partially reversed by washing EGTA containing medium with normal ACSF (19 of 29 cells; three of three slices from three animals) (Fig. 3B). Because the removal of extracellular $\mathrm{Ca}^{2+}$ might deplete intracellular $\mathrm{Ca}^{2+}$ stores, we evaluated the levels of $\mathrm{Ca}^{2+}$ in intracellular stores of astrocytes perfused with nominally $\mathrm{Ca}^{2+}$-free ACSF by activating $\mathrm{IP}_{3}$ and ryanodine receptors. To activate $\mathrm{IP}_{3}$-mediated $\mathrm{Ca}^{2+}$ release from intracellular stores, we used the metabotropic glutamate receptor agonist $t$-ACPD (Porter and McCarthy, 1995). Addition of $t$-ACPD $(5 \mu \mathrm{M})$ to $\mathrm{Ca}^{2+}$-free ACSF triggered a transient increase of $\left[\mathrm{Ca}^{2+}\right]_{\mathrm{i}}$ in 52 of 54 identified astrocytes (two slices from two animals) (Fig. 3C). Moreover, perfusion of the ryanodine receptor agonist 4-CmC (6 mM) (Zorzato et al., 1993; Matyash et al., 2002) in $\mathrm{Ca}^{2+}$-free ACSF elicited a progressive increase of $\left[\mathrm{Ca}^{2+}\right]_{\mathrm{i}}$ in each of the imaged astrocytes (42 astrocytes from two animals) (Fig. $3 D$ ). These results show that intracellular $\mathrm{Ca}^{2+}$ stores are preserved in hippocampal astrocytes perfused with nominally $\mathrm{Ca}^{2+}$-free ACSF and therefore indicate that extracellular $\mathrm{Ca}^{2+}$ is necessary to trigger spontaneous activity in astrocytes.

We next examined whether voltage-gated $\mathrm{Ca}^{2+}$ channels (VGCC) are involved in the spontaneous increase of $\left[\mathrm{Ca}^{2+}\right]_{\mathrm{i}}$ in astrocytes by addition of the nonselective VGCC blocker $\mathrm{Co}^{2+}$. After perfusion of GFAP/GFP hippocampal slices with normal ACSF containing $1 \mathrm{~mm} \mathrm{Co}^{2+}$, only 1 of 32 astrocytes (three slices from three animals) exhibited spontaneous $\left[\mathrm{Ca}^{2+}\right]_{\mathrm{i}}$ oscillations (Fig. $3 A$ ). These data support the notion that spontaneous astrocyte $\mathrm{Ca}^{2+}$ activity requires extracellular $\mathrm{Ca}^{2+}$ and operating voltage-gated $\mathrm{Ca}^{2+}$ channels.

Finally, we analyzed the contribution of released intracellular $\mathrm{Ca}^{2+}$ in spontaneous $\left[\mathrm{Ca}^{2+}\right]_{\mathrm{i}}$ transients of astrocytes by application of thapsigargin, which depletes intracellular $\mathrm{Ca}^{2+}$ stores by inhibiting endoplasmic reticulum $\mathrm{Ca}^{2+}$-ATPase activity (Thastrup et al., 1990). Addition of thapsigargin $(2 \mu \mathrm{M})$ to hippocampal slices decreased the number of astrocytes with spontaneous $\left[\mathrm{Ca}^{2+}\right]_{\mathrm{i}}$ transients (24 of 32 cells; four of four slices from four mice) by $\sim 75 \%$ (Fig. $3 A, B$ ). During thapsi-

\section{$\longleftarrow$}

(Figure legend continued.) squares mark GFP-positive astrocytes, whereas GFP-negative neurons (located mainly in the pyramidal layer) are labeled by white squares. $C$, Representative plots of spontaneous changes of fura-2 fluorescence $(\Delta F / F)$ over 800 sec in astrocytes and neurons in the CA1 hippocampal region. The initiation of each $\left[\mathrm{Ca}^{2+}\right]_{\mathrm{i}}$ oscillation is labeled by a thick mark at the bottom of the plot. $D$, Histograms illustrating properties of spontaneous $\left[\mathrm{Ca}^{2+}\right]_{\mathrm{i}}$ oscillations in astrocytes (white bars) and neurons (black bars). Both the duration and amplitude of spontaneous Ca ${ }^{2+}$ activations are higher in astrocytes than in neurons, whereas the proportion of active cells and the rate of oscillations are similar in both populations. $E$, Raster plot representing the activation profile of each of the 80 active cells shown in $B$ over $800 \mathrm{sec}$. In the raster plot, each active cell is represented by a line, and each thick line marks the initiation of a $\left[\mathrm{Ca}^{2+}\right]_{\mathrm{i}}$ transient. Cells $1-27$ correspond to astrocytes, and $28-80$ correspond to neurons. Dotted lines in the astrocyte raster plot indicate simultaneous coactivation of at least two cells of the plot. Synchronous coactivation among large numbers of neurons can also be seen clearly. $F$, Correlation map illustrating each active astrocyte of $B$ (black squares), in which cells with statistically significant correlation coefficients are connected by lines. The thickness of the lines is proportional to the degree of significance. Most active astrocytes appear to belong to a correlated network. $G$, Correlated map representing synchronous correlations among all the astrocytes (black squares) shown in $F$ and seven representative neurons (white squares, arrows in raster plot). Observe how many astrocytes are synchronously connected with neuronal cells. $H$, Distribution of pairwise correlations found in the real data (arrow) and in 1000 simulations obtained by the Monte Carlo test (bell-shaped curve) of the astrocyte population shown in $B$. Note how the number of correlated events in the real data set (arrow) exceeds those expected by chance in simulated data. The corresponding $p$ value is shown in F. I, Average of Monte Carlo $p$ values showing the probability that the number of times that any two cells had simultaneous onset of activation was caused by chance. Although all cases in both astrocytes $(6$ of $6 ; A)$ and neurons $(6$ of $6 ; N)$ are significant $(p<$ $0.05)$, astrocytic values are higher than neuronal ones. $J$, Average of Monte Carlo $p$ values showing the probability that the number of times the same cells were activated simultaneously at least twice was caused by chance. Each set of neuronal populations gives a $0 p$ value $(6$ of $6 ; N)$, whereas in astrocytic cells only four of six cases are significant $(p<0.05)(A)$. Both $I$ and $J$ illustrate the higher synchronous correlation level of spontaneous $\left[\mathrm{Ca}^{2+}\right]_{\mathrm{i}}$ oscillations among neurons than among astrocytes. $K$, Histograms summarizing the proportion of spontaneous active cells with statistically significant correlation coefficients: among astrocytes $(A \rightarrow A)$, among neurons $(N \rightarrow N)$, percentage of astrocytes coactive with neurons $(A \rightarrow N)$, and percentage of neurons coactive with astrocytes $(N \rightarrow A)$. Statistical significance: ${ }^{*} p<0.0001$. Scale bar, $40 \mu \mathrm{m}$. $s r$, Stratum radiatum; $s p$, stratum pyramidale; $s o$, stratum oriens. 
A
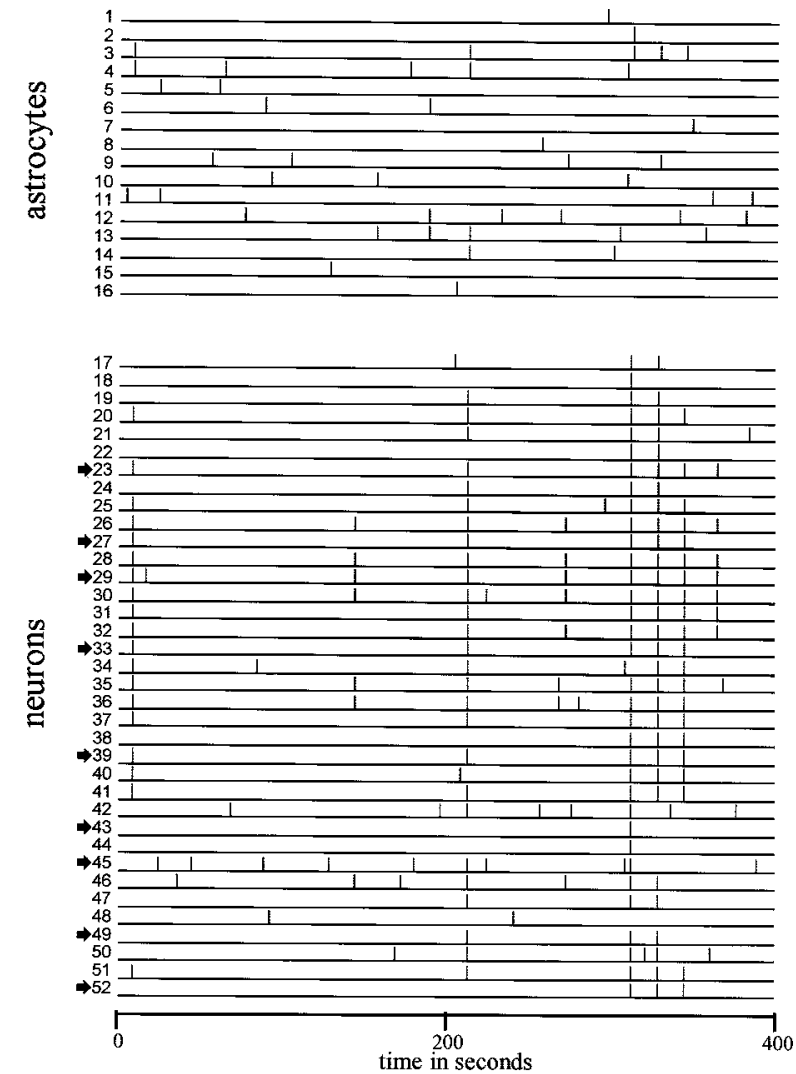

B
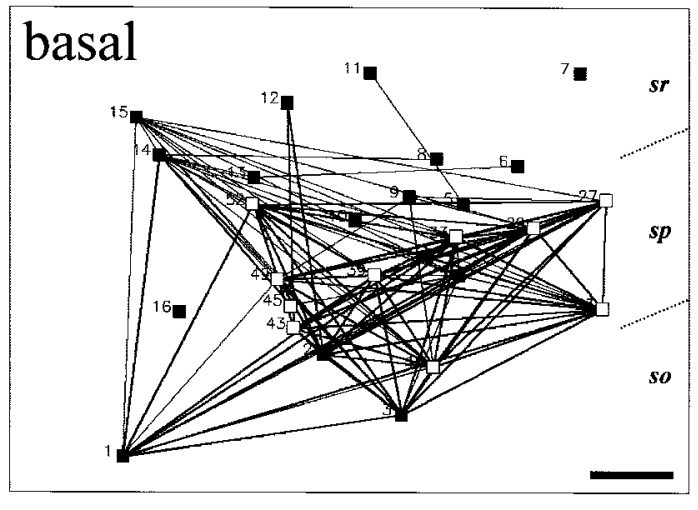

TTX
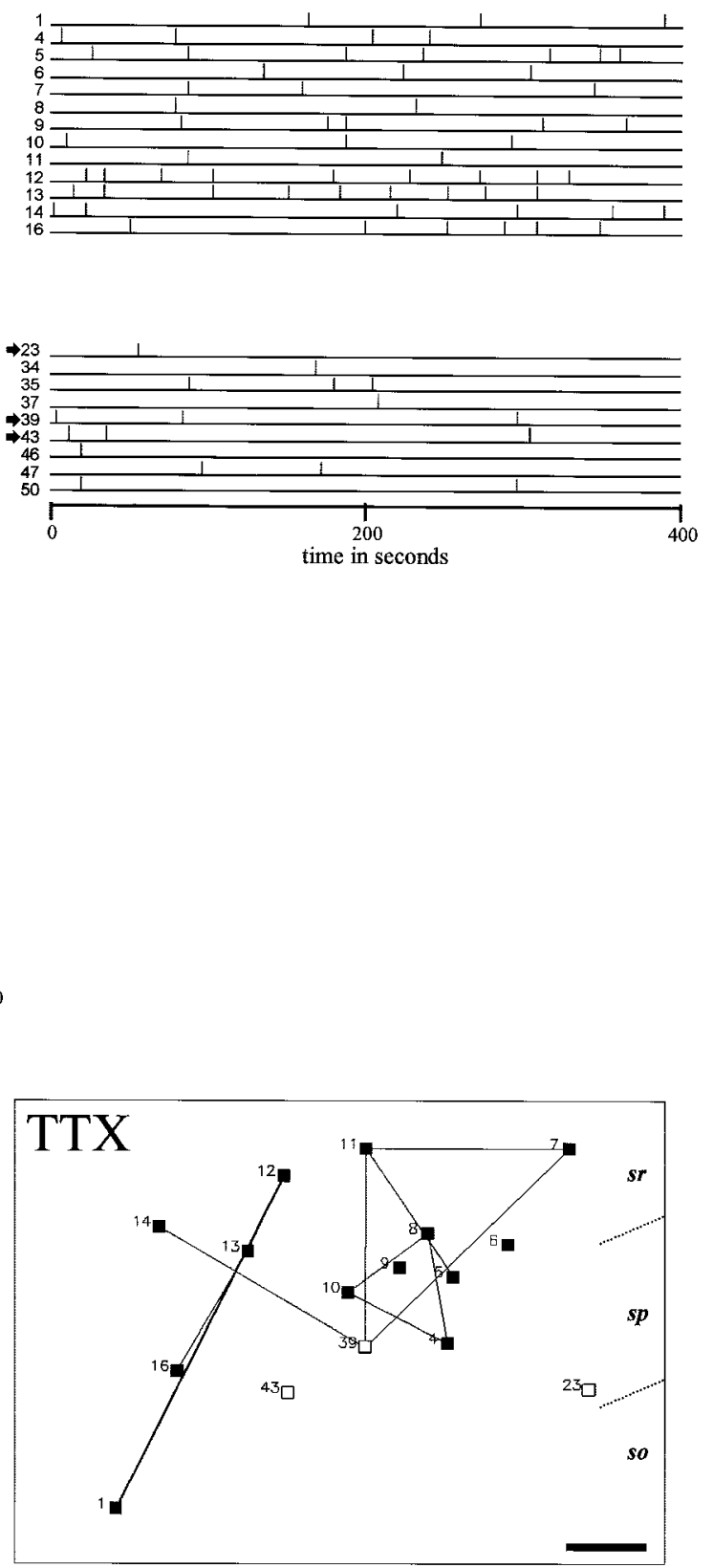

C

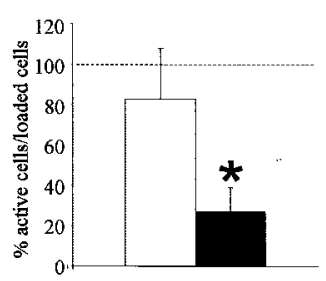

Active cells $D$ Activation rate

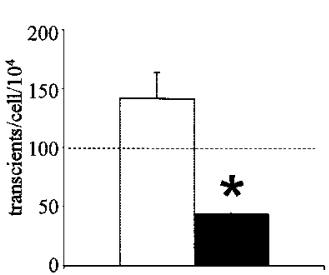

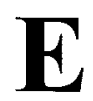

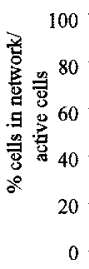

Cells in Network

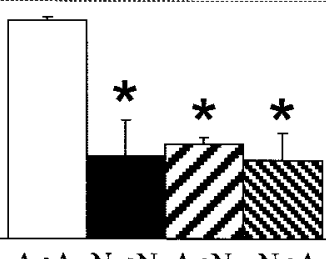

Network correlation

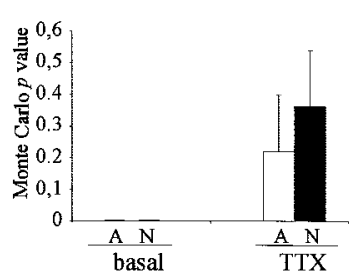


gargin and EGTA treatments, the astrocytes that maintained spontaneous $\mathrm{Ca}^{2+}$ signaling had lower amplitudes and frequencies of oscillation than in basal conditions (data not shown). Moreover, although either nominally $\mathrm{Ca}^{2+}$-free ACSF or $1 \mathrm{~mm} \mathrm{Co}^{2+}$ abolished $\left[\mathrm{Ca}^{2+}\right]_{\mathrm{i}}$ oscillations in virtually all imaged neurons (six slices), neuronal $\left[\mathrm{Ca}^{2+}\right]_{\mathrm{i}}$ transients were mostly preserved $(92.5 \%)$ after addition of $2 \mu \mathrm{M}$ thapsigargin (25 of 27 neurons; two slices). This indicates that in contrast to astrocytes, neuronal oscillations depend almost exclusively on extracellular $\mathrm{Ca}^{2+}$. Taken together, these observations show that extracellular $\mathrm{Ca}^{2+}$ influx and $\mathrm{Ca}^{2+}$ release from intracellular stores are required to generate spontaneous $\left[\mathrm{Ca}^{2+}\right]_{\mathrm{i}}$ transients in hippocampal astrocytes in situ. Moreover, the similarities between these pharmacological findings and those in the thalamus (Parri et al., 2001) suggest that spontaneous astrocytic activity is generated by common mechanisms intrinsic to astrocytes in distinct brain regions.

\section{Astrocytes are integrated in complex spontaneous active networks of neurons and glial cells}

An important feature of spontaneous $\left[\mathrm{Ca}^{2+}\right]_{\mathrm{i}}$ oscillations in developing neurons is the recruitment of active cells into synchronous networks that amplify the functional consequences of activity to control neuronal development (Yuste et al., 1992; Buonanno and Fields, 1999; Feller, 1999; O’Donovan, 1999, Wong, 1999, Ben-Ari, 2001). In our experiments, visual examination of the movies recorded in the neocortex and hippocampus showed synchronous patterns of activity in neuronal populations (Fig. 4), which have been described previously (Ben-Ari et al., 1997; Garaschuk et al., 1998). Interestingly, spatiotemporal correlations of $\mathrm{Ca}^{2+}$ signaling between GFAP/GFP-positive astrocytes were also observed.

Because high numbers of astrocytes and neurons can be accurately identified in GFAP/GFP-positive slices, we analyzed the occurrence of patterned networks of correlated $\mathrm{Ca}^{2+}$ transients among astrocytic and neuronal cells in P5-P7 GFAP/GFPpositive hippocampal slices. First, we compared the astrocytes and neurons present in the same fields for profiles of spontaneous $\left[\mathrm{Ca}^{2+}\right]_{\mathrm{i}}$ transients. As above, astroglial cells were identified by the emission of GFP fluorescence, whereas neurons were identified by the lack of GFP expression and by their morphology and location in the hippocampal layers (Figs. 4A,B). Spontaneous $\left[\mathrm{Ca}^{2+}\right]_{\mathrm{i}}$ changes were monitored simultaneously in dozens of glial (Fig. 4B, $\square$ ) and neuronal (Fig. 4B, $\square$ ) cells, and the onset of each calcium event in every active cell was labeled in graphed plots by a mark (Fig. 4C). No statistical differences were found in the percentages of active cells or in the rates of $\left[\mathrm{Ca}^{2+}\right]_{i}$ oscillations between hippocampal astrocytes and neurons (Fig. 4D), indicating that astrocyte activity is a robust phenomenon. In contrast, both the duration and the amplitude of these oscillations were threefold higher in astrocytes than in neurons (Fig. 4D), as also illustrated in representative plots of glial and neuronal activation profiles (Fig. 4C). This is consistent with the analysis of the fast and slow calcium events in neocortical slices (Badea et al., 2001; Thasiro et al., 2002).

Next we performed a synchrony network analysis among individual astroglial cells to define spatiotemporal patterns of spontaneous $\mathrm{Ca}^{2+}$ oscillations in glial cells. We used a recently developed statistical method that can identify and map the simultaneous coactivations among vast numbers of individual cells (Schwartz et al., 1998; Aguiló et al., 1999). First, we outlined in raster plots the profile of the $\mathrm{Ca}^{2+}$ events of every active cell in each movie (Fig. $4 E$ ). We then used contingency tables and $\chi^{2}$ tests to identify the active cells with significant synchronous $\mathrm{Ca}^{2+}$ transients (Schwartz et al., 1998, Aguiló et al., 1999). This analysis led to correlation maps showing all the active cells recorded from a field in which each pair of synchronous cells is connected by lines the thickness of which is proportional to the degree of correlation (see Materials and Methods) (Figs. 4F, G). Spatiotemporal analysis of astrocytic spontaneous $\left[\mathrm{Ca}^{2+}\right]_{\mathrm{i}}$ changes revealed complex networks of coactivated glial cells (Fig. $4 F$ ). Correlated $\mathrm{Ca}^{2+}$ oscillations were observed between neighboring astrocytes (Fig. $4 F$, e.g., cells 1 and 2 or 10 and 11 ) and also between distant cells (Fig. $4 F$, e.g., cells 1 and 24 or 7 and 24). In the hippocampus, single networks of synchronous astrocytes included cells located in distinct layers, such as the stratum oriens, the stratum radiatum, and the pyramidal layer (Fig. $4 B, F$ ). Quantitative analysis of correlated astroglial maps showed that $\sim 81 \%$ of the active astrocytes belonged to synchronous networks (Fig. $4 K, A \rightarrow A$ ).

We also quantified the overall degree of synchronous correlation in each astroglial network. To calculate the level of coactivation present in each recording, we compared the number of times that any two cells had simultaneous onset activation times in each piece of real data (number of coactivations) with the number of coactivation times present in a distribution of 1000 random experiments created by Monte Carlo simulation (Schwartz et al., 1998) (Fig. 4H). This comparison gives a $p$ value for the real data set that describes the probability that all of the coactivations present in each sample were caused by chance (Fig. $4 H$ ). To standardize the $p$ value of each sample, Monte Carlo simulations were created using the same number of cells, activations, and time intervals as in the real data set, but the initiation of the transients was randomly chosen (see Materials and Methods). Using this analysis, we found an overall significant correlation ( $p<0.05$; Monte Carlo simulation) in six of six astroglial networks imaged from P5-P7 hippocampus (see $p$ value of the network illustrated in Fig. $4 F$ ) with an average $p$ value of 0.002 (Fig. 4I). Taken together, these data show that astrocytic spontaneous $\left[\mathrm{Ca}^{2+}\right]_{\mathrm{i}}$ transients are organized into complex synchro-

\footnotetext{
Figure 5. Spontaneous astrocytic correlated network activity is impaired after TTX treatment. $A$, Raster plot illustrating the activation profiles of astrocytes (cells 1-16) and neurons (cells 17-52) of a hippocampal CA1 field from a P6 GFAP/GFP mouse before (basal) and after TTX administration. Although major changes are not observed in the astrocyte population, neuron activity is greatly impaired. $B$, Correlation maps of all active astrocytes (black squares) and a representative fraction of active neurons (white squares, arrows in raster plots) illustrated in $A$. After TTX treatment active neurons and their correlations are almost absent, whereas correlated astroglial activity persisted. $C$, Spontaneous active astrocytes (white bar) and neurons (black bar) in relation to basal conditions after TTX administration. $D$, Activity rate in astrocytes (white bar) and neurons (black bar) in relation to control after TTX administration. E, Histograms summarizing the proportion of spontaneous active cells with statistically significant correlation coefficients after TTX: among astrocytes $(A \rightarrow A)$, among neurons $(N \rightarrow N)$, percentage of astrocytes coactive with neurons $(A \rightarrow N)$, and percentage of neurons coactive with astrocytes $(N \rightarrow A)$. $F$, Average of Monte Carlo $p$ values showing the probability that the number of times that any two cells had simultaneous onset of activation was caused by chance. Both astrocytes $(A)$ and neurons $(N)$ exhibit very significant values in basal conditions, whereas TTX treatment decorrelates spontaneous activity in both neural populations. Significant reductions $\left({ }^{*} p<0.05\right)$ are observed after TTX. Scale bar, $40 \mu \mathrm{m} . s r$, Stratum radiatum; $s p$, stratum pyramidale; $s o$, stratum oriens.
} 
A
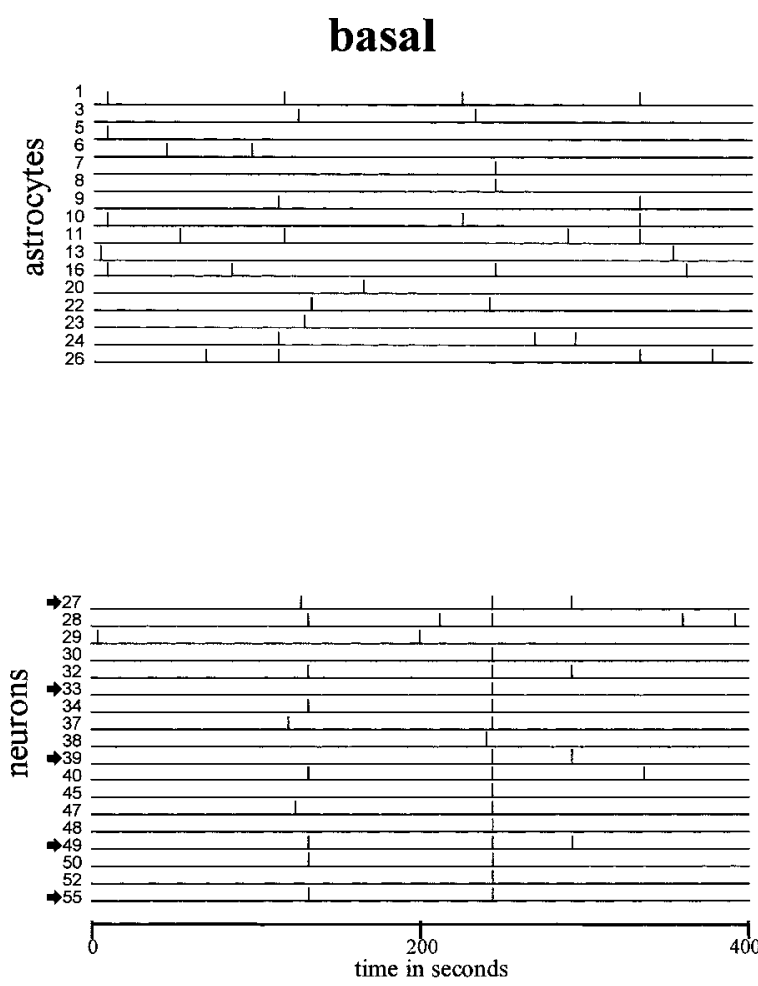

B

\section{basal}

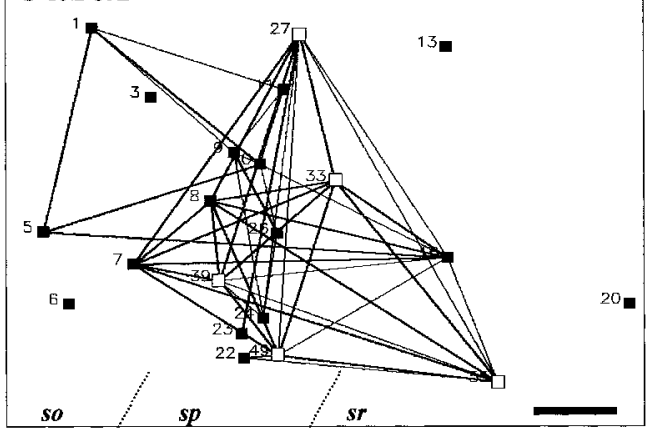

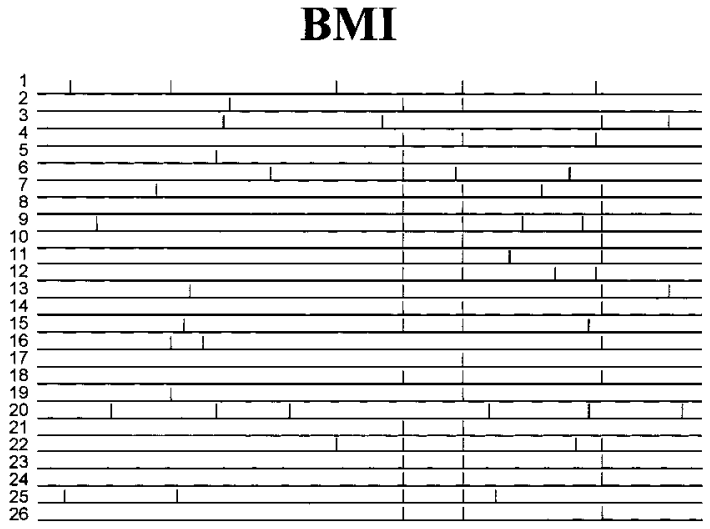
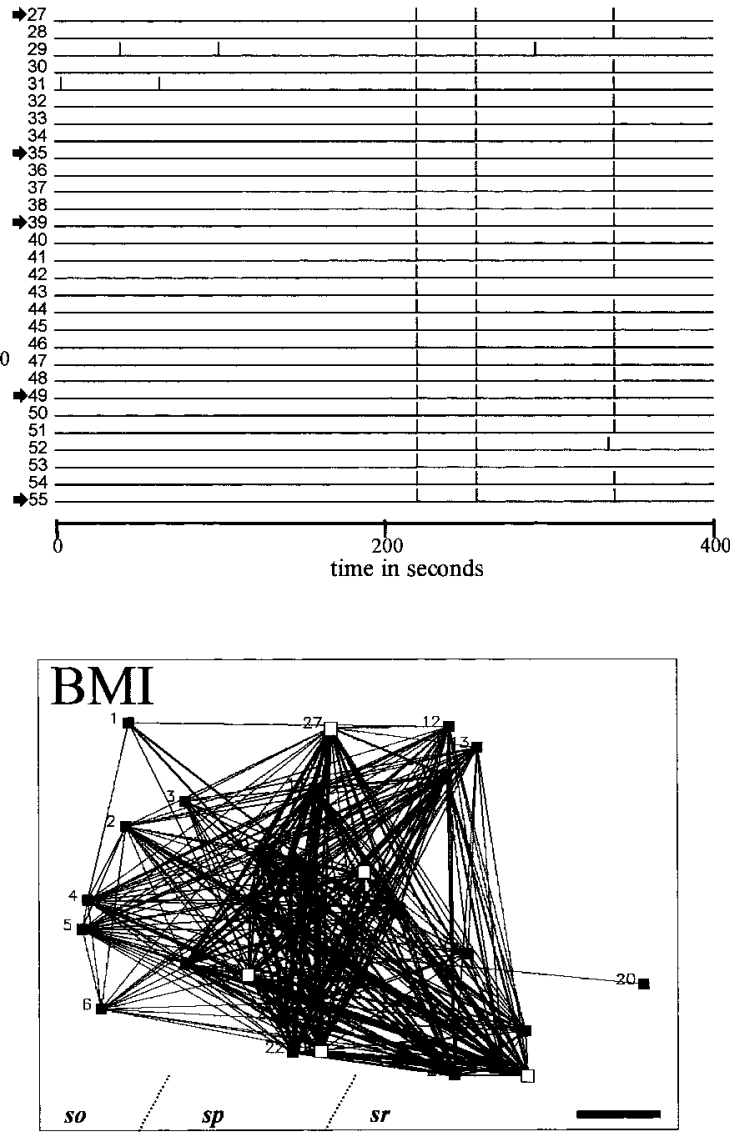

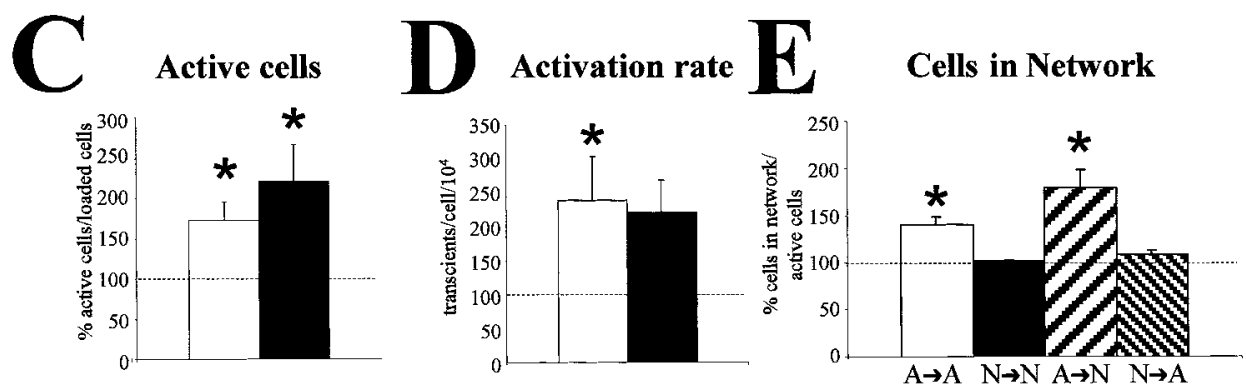

$\mathbf{F}$

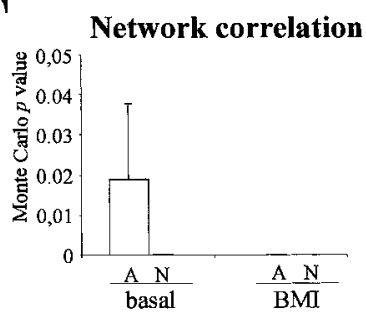

Figure 6. Spontaneous astrocytic correlated network activity increases in BMI-induced epileptiform status. $A$, Raster plot illustrating the activation profiles of astrocytes (cells 1-26) and neurons (cells 27-55) of a hippocampal CA1 field from a P9 GFAP/GFP mouse before (basal) and after BMI administration. BMI enhances spontaneous activity in both astrocytic and neuronal populations. B, Correlation maps showing (Figure legend continues.) 
nous networks that recruit large numbers of astrocytes, which are often located at great distance.

In the imaged movies that analyzed astrocytic $\left[\mathrm{Ca}^{2+}\right]_{\mathrm{i}}$ oscillations, we also observed highly synchronous patterns of spontaneous $\mathrm{Ca}^{2+}$ transients among hippocampal neurons (Fig. 4E, $G$ ). Postnatal hippocampal neurons displayed frequent synchronous events, which corroborated previous studies (Garaschuk et al., 1998, Ben-Ari, 2001). The highly synchronous patterns of hippocampal neuronal network activity were easily observed in raster plots (Fig. 4E) and in correlation maps (Fig. 4G) and recruited most active neurons. In fact, virtually all spontaneous active neurons were synchronously interconnected (Fig. 4E, $G$, and illustrated as $N \rightarrow N$ in $K$ ). Thus, each neuronal network displayed very significant overall synchrony, as shown by the $p$ value 0 of the Monte Carlo test in each sample analyzed (six cases of six) (Fig. $4 I$ ), which indicated that the degree of synchrony was higher in neuronal than in astrocytic networks (compare also the raster plots in Fig. 4E). To confirm this hypothesis, we then used a more restrictive test to compare the number of times that synchronous cells are simultaneously coactivated at least twice in each astroglial and neuronal data set, with 1000 random experiments created by Monte Carlo simulation (Schwartz et al., 1998, Aguiló et al., 1999). $p$ values were higher among astrocytic $(p<0.05$; four of six cases; average $0.078 \pm 12)$ than neuronal networks $(p<$ 0.05 ; six of six cases; average 0$)$, indicating closer correlation in the latter (Fig. $4 J$ ).

Next, we determined possible synchronous correlations of astrocytes and neurons. Examination of mixed glial-neuronal correlation maps showed that subsets of astrocytes and neurons were synchronously interconnected (Fig. 4G). For instance, the active glial cells 1 and 2 were interconnected with neurons 70 and 80 (Fig. 4G). Quantitative analyses of correlation maps showed that $61 \%$ of astrocytes with spontaneous $\left[\mathrm{Ca}^{2+}\right]_{\mathrm{i}}$ transients correlated with spontaneous active neurons (Fig. $4 K, A \rightarrow N$ ). Conversely, most active neurons $(95.4 \%)$ were synchronous with active astrocytes (Fig. $4 K, N \rightarrow A$ ). We conclude that spontaneous correlated network activity is not restricted to neuronal populations, but rather is a common property of developing neurons and astrocytes, which are interconnected into complex synchronous networks.

\section{Network properties of spontaneous astroglial calcium transients are regulated by neuronal activity}

Neuronal activity may be affected by stimulated astroglial $\left[\mathrm{Ca}^{2+}\right]_{\mathrm{i}}$ oscillations (Pasti et al., 1997, 2001; Araque et al., 1998a,b; Bezzi et al., 1998: Parpura and Haydon, 2000; Parri et al., 2001). Conversely, there is evidence that astrocyte-evoked activity may increase after neuronal stimulation (Dani et al., 1992; Porter and McCarthy, 1996; Pasti et al., 1997; Kang et al., 1998). We thus investigated whether neuronal activity regulates spontaneous astrocytic activity and its organization into spatiotemporal correlated networks. First, we used TTX to abolish neuronal action potentials in GFAP/GFP transgenic hippocampal slices (P5-P7).
Incubation with the $\mathrm{Na}^{+}$channel blocker TTX (2 $\left.\mu \mathrm{M}\right)$ impaired spontaneous $\left[\mathrm{Ca}^{2+}\right]_{\mathrm{i}}$ transients in hippocampal neurons (Fig. $5 A$ ). Thus, TTX significantly decreased both the number of neurons showing $\left[\mathrm{Ca}^{2+}\right]_{\mathrm{i}}$ oscillations and the activation rates of the remaining active neurons (Fig. 5C,D). Furthermore, as shown in raster plots and correlation maps, $\mathrm{Na}^{+}$channel blockade dramatically decorrelated the activity profiles of the remaining active neurons, resulting in very simple neuronal networks (Fig. $5 A, B$ ). These observations were supported by a significant reduction in the number of coactive neurons (Fig. $5 E, N \rightarrow N$ ) and by the dramatic rise in the Monte Carlo $p$ value, which reflect the overall network decorrelation (Fig. $5 F$ ).

In contrast, TTX led to slight, although not significant, changes in the number of active astrocytes and their $\left[\mathrm{Ca}^{2+}\right]_{\mathrm{i}}$ oscillation rates (Fig. 5A,C,D). Moreover, although the proportion of interconnected astrocytes was maintained after $\mathrm{Na}^{+}$channel blockade (Fig. $5 E, A \rightarrow A$ ), the patterns of coactivation and overall network synchrony were strikingly impaired, as illustrated by correlation maps (Fig. 5B) and higher Monte Carlo $p$ averages (Fig. $5 F$ ). In addition, the percentage of astrocytes correlated with active neurons decreased significantly after TTX administration (Fig. 5E, $A \rightarrow N)$. Thus, although $\mathrm{Na}^{+}$channels and neuronal activity are not required for the generation of spontaneous astrocyte activity, their correlated network properties with both neurons and astrocytes depend dramatically on action potentials.

To substantiate these findings, we studied whether increased levels of neuronal activity altered spontaneous astrocytic activity. For this, we generated epileptiform discharges in P8-P10 hippocampal slices by administering BMI $(30 \mu \mathrm{M})$ to the bath. Elimination of GABAergic inhibitory neurotransmission by blocking ionotropic $\mathrm{GABA}_{\mathrm{A}}$ receptors by BMI induces hyperexcitability and status epilepticus in cortical slices (Jones and Lambert, 1990; Albowitz et al., 1997; Badea et al., 2001). After BMI application, we observed an enhancement in the number of neurons showing spontaneous $\left[\mathrm{Ca}^{2+}\right]_{\mathrm{i}}$ transients and in their rates of oscillations (Fig. 6 $A, C, D$ ). Moreover, most of the active neurons were recruited into repetitive synchronous waves, which corroborated previous studies (Albowitz et al., 1997; Badea et al., 2001), and led to highly coactive raster plots and correlation maps (Fig. $6 A, B)$. Interestingly, the number of spontaneously active GFAP/ GFP-positive astrocytes and their activation rates also increased dramatically after $\mathrm{GABA}_{\mathrm{A}}$ receptor blockade (Fig. 5A,C,D). More importantly, the number of astrocytes coactive with other astrocytes $(\mathrm{A} \rightarrow \mathrm{A})$ and with neurons $(\mathrm{A} \rightarrow \mathrm{N})$ after BMI incubation increased by 41 and $80 \%$, respectively (Fig. $6 E$ ), which resulted in very complex correlation maps (Fig. 6B) and in high overall astrocyte synchrony (Fig. $6 F$ ). Neuronal epileptiform waves recruited many astrocytic $\left[\mathrm{Ca}^{2+}\right]_{\mathrm{i}}$ oscillations, so that most active astrocytes were synchronous with neuronal activation waves (see raster plot and correlation map in Fig. 6). Thus, BMI treatment not only increased spontaneous astrocytic activity but

\section{$\leftarrow$}

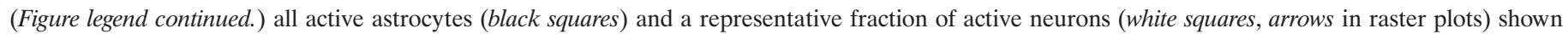

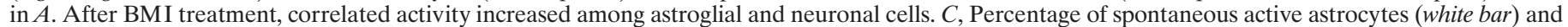

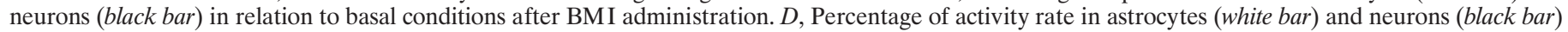

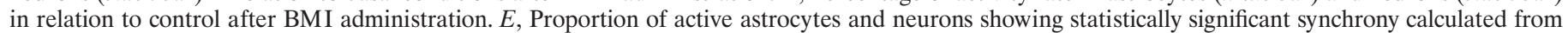

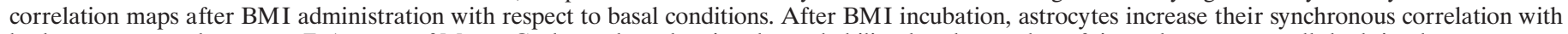

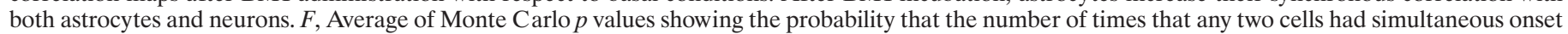

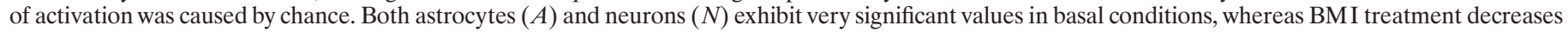

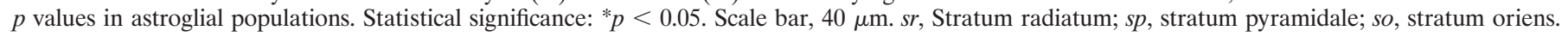



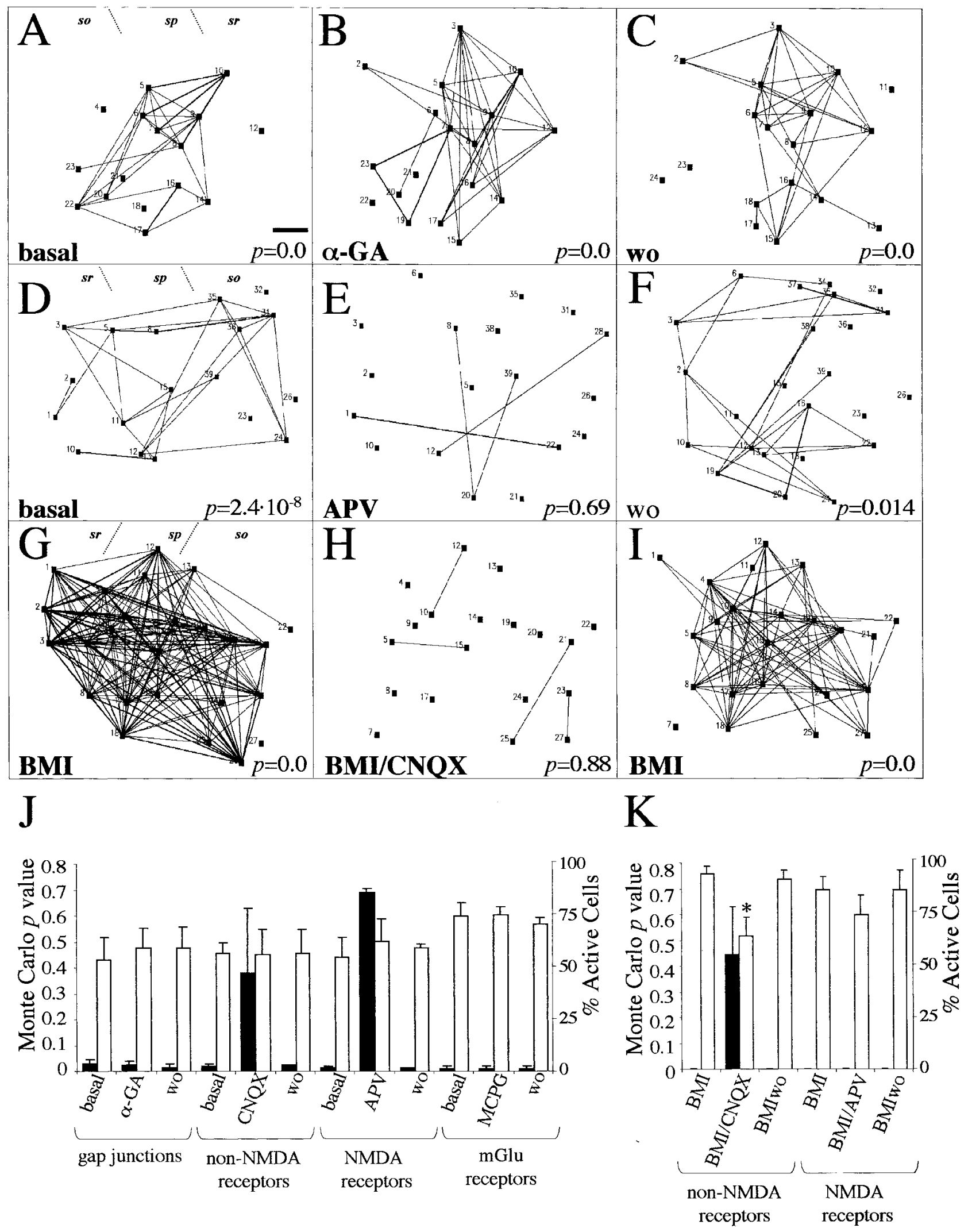

Figure 7. Mechanisms that control network correlation of spontaneous astrocytic activity in the hippocampus. $A-C$, Correlation maps showing coordinated activity of a P6 astrocyte network at basal conditions $(A)$, after incubation with the gap junction blocker $\alpha$-GA $(B)$, and after washout (wo) with $\alpha$-GA $(C)$. Note that the network correlation, indicated by Monte Carlo $p$ value (bottom of the map), is not perturbed by blocking gap junctions. $D-F$, Correlation maps showing the effect of NMDA receptor blockade on a P5 astrocyte network. Correlated Ca ${ }^{2+}$ events in nontreated astrocytes $(D)$ are reduced by APV incubation $(E)$ and recovered after washing the antagonist $(F)$. $G-I$, Network astrocyte correlation in a BMI-treated hippocampal slice $(G)$ is markedly decreased by addition of CNQX $(H)$ and restored by washing the non-NMDA antagonist $(I)$. J, (Figure legend continues.) 
also increased correlated activity between astrocytes and between astrocytes and neurons.

Overall, our results show that although the generation of spontaneous astrocyte activity does not require neuronal activity, its network properties are controlled, to a large extent, by neuronal excitation.

\section{Activation of ionotropic glutamate receptors is required for the generation of correlated astrocytic network activity}

Gap junctions and extracellular messengers, such as glutamate, ATP, and nitric oxide, have been proposed to control the propagation of evoked $\mathrm{Ca}^{2+}$ activation in astrocyte networks (Cornell-Bell et al., 1990; Dani et al., 1992; Finkbeiner, 1992; Venance et al., 1997; Guthrie et al., 1999; Willmott et al., 2000; Newman, 2001; Schipke et al., 2002). To identify the mechanisms that correlate spontaneous activity of astrocytic networks in situ, we used several blocking agents on P5-P7 GFAP/GFP hippocampal slices. Incubation with the gap junction blocker $\alpha$-GA (100 $\mu \mathrm{M}$ ) did not perturb the number of active astrocytes (Figs. $3 A$, $7 A-C, J)$. Moreover, the $p$ values reflecting the correlation degree among the entire astrocyte population were totally preserved (Fig. 7 $A-C, J)$. Next, and because neuronal activity was necessary to correlate spontaneous astrocyte $\mathrm{Ca}^{2+}$ activation, we analyzed the contribution of glutamate receptors. Addition of AMPA/ kainate receptor antagonists $(20 \mu \mathrm{M}$ CNQX) to the bath markedly impaired astrocyte network correlation (Fig. 7J). This effect was reverted by washing out the CNQX (Fig. 7J). Moreover, perfusion with the NMDA receptor antagonist APV $(50 \mu \mathrm{M})$ also abolished astrocytic network correlation (Fig. $7 D, E, J)$. Again, astrocyte coactivations were restored after washout (Fig. $7 F$ ). In contrast, the blockage of glutamate metabotropic receptors by MCPG (1 mM) did not decorrelate spontaneous astrocytic network activity (Fig. $7 J$ ). We conclude that activation of ionotropic glutamate receptors is not necessary for the generation of spontaneous astrocytic activity in situ, but that this activation is a major regulator of their spatiotemporal coordination into correlated networks. Moreover, most of the blocking agents only slightly altered the pattern of spontaneous $\left[\mathrm{Ca}^{2+}\right]_{i}$ oscillations in neurons (data not shown), whereas APV treatment reduced the number of active neurons by $36.8 \%$, in agreement with previous reports (Ben-Ari et al., 1997, Garaschuk et al., 1998).

It is known that the lack of inhibitory $\mathrm{GABA}_{\mathrm{A}}$-mediated responses in BMI-treated hippocampal slices markedly increases neuronal excitability and glutamate release, which in turn produces epileptogenesis (Bradford, 1995). We analyzed the contribution of ionotropic glutamate receptors in the generation of astrocytic network correlation in the BMI-induced epilepsy model. Administration of BMI to P8-P11 GFAP/GFP hippocampal slices increased correlated astrocytic activity (Figs. 6, 7G,K). Addition of CNQX $(20 \mu \mathrm{M})$ to BMI-ACSF dramatically abolished the correlation of astrocytic $\mathrm{Ca}^{2+}$ activity (Fig. $7 G, H, K$ ). This blockage was reverted by washing out the glutamate antagonist with BMI-ACSF (Fig. 7I,K). The decorrelation effect of CNQX on BMI-treated astrocytic networks was accompanied by a significant $37.2 \%$ decrease in the number of spontaneously active astrocytes $(22 \pm 2.7$ vs $14.5 \pm 1.5$ active astrocytes; four slices; $p<0.05$; paired t test) (Fig. $7 G, H, K$ ). In contrast, blockade of NMDA receptors in BMI-treated hippocampal slices did not alter the correlation degree of astroglial networks (Fig. $7 K$ ). Moreover, the number of astrocytes exhibiting $\left[\mathrm{Ca}^{2+}\right]_{\mathrm{i}}$ changes in BMI-ACSF $(21 \pm 3.8$ active astrocytes; three slices) was not perturbed by APV $(18 \pm 2.5$ active astrocytes; three slices; $p=$ 0.46 ; paired $t$ test) (Fig. $7 K$ ). Similarly, incubation of BMIinduced epileptic slices with CNQX decreased by $75.6 \pm 8 \%$ the number of active neurons ( $p<0.05$; paired $t$ test), whereas incubation with APV did not alter the number of active neurons ( $p=0.28$; paired $t$ test). These data show that in conditions of neuronal hyperexcitability, such as epilepsy, the correlation of spontaneous astrocytic network activity is regulated mainly by AMPA/kainate receptors.

\section{DISCUSSION}

Spontaneous $\left[\mathrm{Ca}^{2+}\right]_{\mathrm{i}}$ oscillations in astrocytes have been described in dissociated and organotypic cultures (Fatatis and Rusell, 1992; Charles, 1994; Harris-White et al., 1998). More recently, spontaneous $\left[\mathrm{Ca}^{2+}\right]_{\mathrm{i}}$ transients have been reported in situ in microdomains of cerebellar Bergmann glia surrounding parallel fiber synapses and in the cell bodies of thalamic and hippocampal astrocytes (Grosche et al., 1999; Parri et al., 2001; Nett et al., 2002). Here, we show that astrocytes from a number of brain regions, including the cerebral cortex, display spontaneous activity in situ in all the developmental stages studied and in the adult. The finding that patterns of astrocyte activation were variable, ranging from random events to bursting activity, raises the possibility of correlations with development or with regionspecific patterns of neuronal activity.

Although resting astrocytes are related to the modulation of synaptic transmission (Araque et al., 2001; Bezzi and Volterra, 2001), activated astrocytes play essential roles in injury-related processes such as the healing of scars, repair of the extracellular matrix, removal of debris, and control of the blood-CNS interface (Raivich et al., 1999). Thus, the finding that reactive astrocytes lack $\left[\mathrm{Ca}^{2+}\right]_{\mathrm{i}}$ transients not only demonstrates that spontaneous astrocytic activity depends on the functional state of these cells, but indicates a relationship with the regulation of neural transmission. Because changes in $\left[\mathrm{Ca}^{2+}\right]_{\mathrm{i}}$ control gene expression and cell differentiation (Spitzer et al., 2000), the lack of spontaneous $\left[\mathrm{Ca}^{2+}\right]_{\mathrm{i}}$ transients in astrocytes might contribute to the activation of astrocytes. Taken together, the above data show that spontaneous $\left[\mathrm{Ca}^{2+}\right]_{\mathrm{i}}$ oscillations are a common property of most brain astrocytes that is lost when they become activated after injury.

Although certain neurotransmitters, such as glutamate and ATP, trigger increases in $\left[\mathrm{Ca}^{2+}\right]_{\mathrm{i}}$ and oscillations (Cornell-Bell et al., 1990; Charles et al., 1991; Parpura et al., 1994; Pasti et al., 1997; Porter and McCarthy, 1997; Reetz et al., 1997; Verkhratsky et al., 1998; Guthrie et al., 1999), our results show that the generation of spontaneous activity in hippocampal astrocytes in

\section{$\leftarrow$}

(Figure legend continued.) Histogram showing the average of Monte Carlo $p$ values (black bars, left) and the percentage of active astrocytes (white bars, right) after addition of gap junction blockers and glutamate receptor antagonists in basal GFAP/GFP hippocampal slices. $K$, Average of astrocyte network correlations (black bars, left) and percentage of active astrocytes (white bars, right) after administration of ionotropic glutamate receptor antagonists in BMI-treated hippocampal slices. Each experimental condition was performed in at least three different slices. Statistical significance: ${ }^{*} p<0.05$. Scale bar, $45 \mu \mathrm{m}$. $s r$, Stratum radiatum; $s p$, stratum pyramidale; so, stratum oriens. 
situ is mostly independent of both ATP signaling and activation of glutamatergic and GABAergic receptors, and of neuronal activity, which is consistent with recent reports (Parri et al., 2001; Nett et al., 2002; Thasiro et al., 2002). Moreover, we show that spontaneous oscillations are critically dependent on extracellular and intracellular $\mathrm{Ca}^{2+}$, which might cause the typical large and long-lasting profiles of $\left[\mathrm{Ca}^{2+}\right]_{i}$ oscillations of astrocytes. Thus, it is conceivable that the removal of extracellular $\mathrm{Ca}^{2+}$ may abolish a $\mathrm{Ca}^{2+}$ influx-dependent baseline release of neurotransmitters from nerve terminals that is necessary to activate astroglial cells. However, treatment with the selective V-ATPase inhibitor bafilomycin A1, which inhibits vesicular neurotransmitter release (Araque et al., 2000; Zhou et al., 2000), does not prevent astrocyte $\left[\mathrm{Ca}^{2+}\right]_{\mathrm{i}}$ oscillations in situ (Nett et al., 2002), suggesting a direct requirement of $\left[\mathrm{Ca}^{2+}\right]_{0}$ for the generation of spontaneous astrocytic activity. Finally, the strikingly similar pharmacological profiles observed for astrocytic $\left[\mathrm{Ca}^{2+}\right]_{\mathrm{i}}$ oscillations in the thalamus (Parri et al., 2001) and hippocampus (present results) support the notion that spontaneous astrocytic activity is triggered by mechanisms common to several brain regions.

The finding that the percentage of spontaneously active cells and their oscillation rates are very similar in astrocytes and neurons indicates that astrocytic activity is a robust phenomenon. Using an approach that allows the monitoring of spontaneous $\left[\mathrm{Ca}^{2+}\right]_{\mathrm{i}}$ changes in large numbers of astrocytes, we demonstrate large and complex networks of correlated, synchronous astrocytes. We found that $\sim 80 \%$ of spontaneously active astrocytes in the hippocampus display synchronous astrocyte-to-astrocyte $\left[\mathrm{Ca}^{2+}\right]_{\mathrm{i}}$ events, forming complex networks that recruit dozens of astrocytes, often located at great distances. Interestingly, the patterns of spontaneous coactivation described here in the hippocampus in situ are much more elaborate than those observed previously in dissociated, organotypic cultures and neocortical slices (Dani et al., 1992; Charles, 1998; Harris-White et al., 1998; Thasiro et al., 2002). Similarly, in the thalamus in situ, Parri et al. (2001) described spontaneous synchronous correlations among up to five neighboring astrocytes. The highly synchronous activation characteristic of postnatal hippocampal neurons (Ben-Ari et al., 1997; Garaschuk et al., 1998; Ben-Ari, 2001) might account for these differences in network complexity. However, we favor the view that it is the combined transgenic/imaging approach used in this study, designed to screen activity in large numbers of astrocytes, that has allowed the detection of these complex correlated networks. Finally, because astrocytic networks occur at distinct postnatal stages in the hippocampus (present data), the thalamus (Parri et al., 2001), and other areas, such as the neocortex (F. Aguado, J. F. Espinosa-Parrilla, and E. Soriano, unpublished observations), network activity may be a property of most astrocytes in vivo.

In our experiments, many spontaneously active astrocytes (61\%) had $\mathrm{Ca}^{2+}$ events correlated with hippocampal neurons, which suggests a relationship between spontaneous neuronal and glial activities. In fact, previous studies have described structural and functional interactions between neurons and astrocytes, such as gap junctions and extracellular messengers (Dani et al., 1992; Porter and McCarthy, 1996; Pasti et al., 1997, 2001; Araque et al., 1998a,b; Ventura and Harris, 1999; Alvarez-Maubecin et al., 2000; Rouach et al., 2000; Rochon et al., 2001). Although the percentage of active astrocytes and their rate of oscillation does not change substantially after action potential blockade with TTX, the overall synchronous activity of astrocytic networks in the postnatal hippocampus is abolished, indicating that neuronal activity is required to adjust the spatiotemporal pattern and synchrony of spontaneously active astrocytes. Conversely, greater neuronal activity levels caused by epileptiform discharges with BMI not only raise the number of active astrocytes (Tashiro et al., 2002), but dramatically increases the network correlation between astrocytes and astrocytes and neurons. In fact, in the bicuculline model of epilepsy, many astrocytes are recruited into the highly synchronous epileptiform waves induced in hippocampal neurons, demonstrating a fast spatiotemporal control of glial spontaneous activity by neurons. Thus, astrocyte oscillations and their degree of coactivation are regulated by neurons when the levels of neuronal activity exceed a threshold, as in the epileptiform state.

Gap junctions control $\mathrm{Ca}^{2+}$ wave propagation in cultured astrocytes (Giaume and McCarthy, 1996; Charles, 1998; Giaume and Venance, 1998). The present study, which shows that correlation of spontaneous astrocytic $\mathrm{Ca}^{2+}$ activity is independent of gap junctions, together with the fact that evoked $\mathrm{Ca}^{2+}$ waves in acute brain slices are unaffected by gap junction blockers (Schipke et al., 2001), suggests that different mechanisms correlate $\mathrm{Ca}^{2+}$ activity in cultured and in situ astrocytes. In contrast, inhibition of NMDA and non-NMDA glutamate receptors impairs spontaneous astrocytic activity, which is consistent with other studies that show that activation of glutamate receptors is involved in the propagation of astrocyte $\mathrm{Ca}^{2+}$ signaling (Cornell-Bell et al., 1990; Cornell-Bell and Finkbeiner, 1991; Dani et al., 1992; Finkbeiner, 1992; Venance et al., 1997). Because, in addition to neurons, astrocytes exhibit $\mathrm{Ca}^{2+}$-dependent glutamate release (Bezzi et al., 1998, Araque et al., 2000; Innocenti et al., 2000), astrocytic network activity could be controlled by glutamate derived from both neurons and astrocytes. It is noteworthy that TTX and ionotropic glutamate receptor antagonists decorrelate astrocytic networks in a similar manner, without altering the number of active astrocytes. These observations indicate that glutamate released from depolarized nerve terminals contributes to the correlation of astrocyte $\mathrm{Ca}^{2+}$ activity. This view is also in agreement with data on the functional expression of NMDA and non-NMDA receptors in astrocytes (Porter and McCarthy, 1995; Schipke et al., 2001).

We also show that CNQX, but not APV, reverts the BMIinduced increased levels of spontaneous activity of astrocytes and their spatiotemporal coordination. Because activation of AMPA/ kainite but not NMDA receptors is the main mechanism involved in the generation and propagation of epileptiform discharges in BMI-treated hippocampus (Jones and Lambert, 1990; Albowitz et al., 1997; Stoop and Pralong, 2000), the view that neuronalreleased glutamate correlates astrocytic network activity is supported further. Taken together, the present data indicate that neuronal glutamate modulates the network properties of spontaneous astrocytic $\mathrm{Ca}^{2+}$ signaling by activating ionotropic receptors.

In summary, we conclude that $\left[\mathrm{Ca}^{2+}\right]_{\mathrm{i}}$ oscillations in astrocytes and their network properties are generated by intrinsic mechanisms in vivo, which are modulated, however, by neuronal activity and fast glutamate receptors. Thus, together with data showing that astrocyte stimulation leads to neuronal discharges (Pasti et al., 1997, 2001; Araque et al., 1998a,b; Bezzi et al., 1998; Parpura and Haydon, 2000; Parri et al., 2001), our results point to a complex functional scenario in which spontaneous astrocyte and neuronal activities are regulated bi-directionally in a dynamic manner. Given the essential role of spontaneous and evoked neuronal activity in diverse events during neuronal development 
and in synaptic plasticity (Katz and Shatz, 1996), a complex neuronal/astrocytic network may play a key role in these processes. In addition, our data point to an unexplored function of astrocyte $\left[\mathrm{Ca}^{2+}\right]_{\mathrm{i}}$ signaling in neuropathological processes such as epilepsy.

\section{REFERENCES}

Aguiló A, Schwartz TH, Kumar VS, Peterlin ZA, Tsiola A, Soriano E, Yuste R (1999) Involvement of Cajal-Retzius neurons in spontaneous correlated activity of embryonic and postnatal layer 1 from wild-type and reeler mice. J Neurosci 19:10856-10868.

Albowitz B, König P, Kuhnt U (1997) Spatiotemporal distribution of intracellular calcium transients during epileptiform activity in guinea pig hippocampal slices. J Neurophysiol 77:491-501.

Alvarez-Maubecin V, García-Hernández F, Williams JT, van Bockstaele EJ (2000) Functional coupling between neurons and glia. J Neurosci 20:4091-4098.

Araque A, Parpura V, Sanzgiri RP, Haydon PG (1998a) Glutamatedependent astrocyte modulation of synaptic transmission between cultured hippocampal neurons. Eur J Neurosci 10:2129-2142.

Araque A, Sanzgiri RP, Parpura V, Haydon PG (1998b) Calcium elevations in astrocytes cause an NMDA receptor-dependent increase in the frequency of miniature synaptic currents in cultured hippocampal neurons. J Neurosci 18:6822-6829.

Araque A, Li N, Doyle RT, Haydon PG (2000) SNARE proteindependent glutamate release from astrocytes. J Neurosci 20:666-673.

Araque A, Carmignoto G, Haydon PG (2001) Dynamic signaling between astrocytes and neurons. Annu Rev Physiol 63:795-813.

Badea T, Goldberg J, Mao BQ, Yuste R (2001) Calcium imaging of epileptiform events with single-cell resolution. J Neurobiol 48:215-227.

Ben-Ari Y (2001) Developing networks play a similar melody. Trends Neurosci 24:353-360.

Ben-Ari Y, Khazipov R, Leinekugel X, Caillard O, Gaiarsa JL (1997) $\mathrm{GABA}_{\mathrm{A}}$, NMDA and AMPA receptors: a developmentally regulated "ménage à trois." Trends Neurosci 20:523-529.

Berridge MJ (1998) Neuronal calcium signaling. Neuron 21:13-26.

Bezzi P, Volterra A (2001) A neuron-glia signalling network in the active brain. Curr Opin Neurobiol 11:387-394.

Bezzi P, Carmignoto G, Pasti L, Vesce S, Rossi D, Rizzini BL, Pozzan T, Volterra A (1998) Prostaglandins stimulate calcium-dependent glutamate release in astrocyte. Nature 391:281-285.

Bradford HF (1995) Glutamate, GABA and epilepsy. Prog Neurobiol 47:477-511.

Buonanno A, Fields RD (1999) Gene regulation by patterned electrical activity during neural and skeletal muscle development. Curr Opin Neurobiol 9:110-120.

Carmignoto G (2000) Reciprocal communication systems between astrocytes and neurons. Prog Neurobiol 62:561-581.

Charles A (1998) Intercellular calcium waves in glia. Glia 24:39-49.

Charles AC (1994) Glia-neuron intercellular calcium signaling. Dev Neurosci 16:196-206.

Charles AC, Merill JE, Dirksen ER, Sanderson MJ (1991) Intercellular signaling in glial cells: calcium waves and oscillations in response to mechanical stimulation and glutamate. Neuron 6:983-992.

Cooper MS (1995) Intercellular signaling in neuronal-glial networks. BioSystems 34:65-85.

Cornell-Bell AH, Finkbeiner SM (1991) $\mathrm{Ca}^{2+}$ waves in astrocytes. Cell Calcium 12:185-204.

Cornell-Bell AH, Finkbeiner SM, Cooper MS, Smith SJ (1990) Glutamate induces calcium waves in cultured astrocytes: long-range glial signaling. Science 247:470-473.

Cunha RA, Ribeiro JA (2000) ATP as a presynaptic modulator. Life Sci 68:119-137.

Dani JW, Chernjavsky A, Smith SJ (1992) Neuronal activity triggers calcium waves in hippocampal astrocyte networks. Neuron 8:429-440.

Davidson JS, Baumgarten IM (1998) Glycyrrhetinic acid derivatives: a novel class of inhibitors of gap-junctional intercellular communication. Structure-activity relationships. J Pharmacol Exp Ther 246:1104-1107.

Fatatis A, Rusell JT (1992) Spontaneous changes in intracellular calcium concentration in type I astrocytes from rat cerebral cortex in primary culture. Glia 5:95-104.

Feller MB (1999) Spontaneous correlated activity in developing neural circuits. Neuron 22:653-656.

Fields RD, Stevens B (2000) ATP: an extracellular signaling molecule between neurons and glia. Trends Neurosci 23:625-633.

Finkbeiner S (1992) Calcium waves in astrocytes-filling in the gaps. Neuron 8:1101-1108.

Garaschuk O, Hanse E, Konnerth A (1998) Developmental profile and synaptic origin of early network oscillations in the CA1 region of rat neonatal hippocampus. J Physiol (Lond) 507.1:219-236.

Giaume C, McCarthy KD (1996) Control of junctional communication in astrocytic networks. Trends Neurosci 19:319-325.
Giaume C, Venance L (1998) Intercellular calcium signaling and gap junctional communication in astrocytes. Glia 24:50-64.

Grosche J, Matyash V, Möller T, Verkhratsky A, Reichenbach A, Kettenmann H (1999) Microdomains for neuron-glia interaction: parallel fiber signaling to Bergmann glial cells. Nat Neurosci 2:139-143.

Guthrie PB, Knappenberger J, Segal M, Bennett MV, Charles CA, Kater SB (1999) ATP released from astrocytes mediates glial calcium waves. J Neurosci 19:520-528.

Harris-White ME, Zanotti SA, Frautschy SA, Charles AC (1998) Spiral intercellular calcium waves in hippocampal slice cultures. J Neurophysiol 79:1045-1052.

Haydon PG (2001) Glia: listening and talking to the synapse. Nat Rev 2:185-193.

Innocenti B, Parpura V, Haydon PG (2000) Imaging extracellular waves of glutamate during calcium signaling in cultured astrocytes. J Neurosci 20:1800-1808

Jones RS, Lambert JD (1990) The role of excitatory amino acids in the propagation of epileptiform discharges from the entorhinal cortex to the dentate gyrus in vitro. Exp Brain Res 80:310-322.

Kang J, Jiang L, Goldman SA, Nedergaard M (1998) Astrocytemediated potentiation of inhibitory synaptic transmission. Nat Neurosci 1:683-692.

Katz LC, Shatz CJ (1996) Synaptic activity and the construction of cortical circuits. Science 274:1133-1138.

Kettenmann H, Ransom BR (1995) Neuroglia. New York: Oxford UP.

Komuro H, Rakic O (1998) Orchestration of neuronal migration by activity of ion channels, neurotransmitter receptors, and intracellular $\mathrm{Ca}^{2+}$ fluctuations. J Neurobiol 37:110-130.

Latov N, Nilaver G, Zimmerman EA, Johson WG, Silverman AJ, Defendini R, Cote L (1979) Fibrillary astrocytes proliferate to brain injury. Dev Biol 72:381-384.

Mathewson AJ, Berry M (1985) Observations on the astrocyte response to a cerebral stab wound in adult rats. Brain Res 327:61-69.

Matyash M, Matyash V, Nolte C, Sorrentino V, Kettenmann H (2002) Requirement of functional ryanodine receptor type 3 for astrocyte migration. FASEB J 16:84-86.

Nett WJ, Oloff SH, McCarthy KD (2002) Hippocampal astrocytes in situ exhibit calcium oscillations that occur independent of neuronal activity. J Neurophysiol 87:528-537.

Newman EA (2001) Propagation on intercellular calcium waves in retinal astrocytes and Müller cells. J Neurosci 21:2215-2223.

Nolte C, Matyash M, Pivneva T, Schipke CG, Ohlemeyer C, Hanisch UK, Kirchhoff F, Kettenmann H (2001) GFAP promoter-controlled EGFP-expressing transgenic mice: a tool to visualize astrocytes and astrogliosis in living brain tissue. Glia 33:72-86.

O'Donovan MJ (1999) The origin of spontaneous activity in developing networks of the vertebrate nervous system. Curr Opin Neurobiol 9:94-104.

Parpura V, Haydon PG (2000) Physiological astrocytic calcium levels stimulate glutamate release to modulate adjacent neurons. Proc Natl Acad Sci USA 97:8629-8634.

Parpura V, Basarky TA, Liu F, Jeftinija K, Jeftinija S, Haydon PG (1994) Glutamate-mediated astrocyte-neuron signalling. Nature 369:744-747.

Parri HR, Gould TM, Crunelli V (2001) Spontaneous astrocytic $\mathrm{Ca}^{2+}$ oscillations in situ drive NMDA-mediated neuronal excitation. Nat Neurosci 4:803-812.

Pasti L, Volterra A, Pozzan T, Carmignoto G (1997) Intracellular calcium oscillations in astrocytes: a highly plastic, bi-directional form of communication between neurons and astrocytes in situ. J Neurosci 17:7817-7830.

Pasti L, Zonta M, Pozzan T, Vicini S, Carmignoto G (2001) Cytosolic calcium oscillations in astrocytes may regulate exocytotic release of glutamate. J Neurosci 15:477-484.

Porter JT, McCarthy KD (1995) GFAP-positive hippocampal astrocytes in situ respond to glutamatergic neuroligands with increases in $\left[\mathrm{Ca}^{2+}\right]_{\mathrm{i}}$. Glia $13: 101-112$.

Porter JT, McCarthy KD (1996) Hippocampal astrocytes in situ respond to glutamate released from synaptic terminals. J Neurosci 16:5073-5081.

Porter JT, McCarthy KD (1997) Astrocytic neurotransmitter receptors in situ and in vivo. Prog Neurobiol 51:439-455.

Raivich G, Bohatschek M, Kloss CUA, Werner A, Jones LL, Kreutzberg GW (1999) Neuroglial activation repertoire in the injured brain: graded response, molecular mechanisms and cues to physiological function. Brain Res Rev 30:77-105.

Reetz G, Wiesinger H, Reiser G (1997) ATP-induced oscillations of cytosolic $\mathrm{Ca}^{2+}$ activity in cultured astrocytes from rat brain are modulated by medium osmolarity indicating a control of $\left[\mathrm{Ca}^{2+}\right]_{\mathrm{i}}$ oscillations by cell volume. Neurochem Res 22:621-628.

Rochon D, Rousse I, Robitalle R (2001) Synapse-glia interactions at the mammalian neuromuscular junction. J Neurosci 21:3819-3829.

Rouach N, Glowinski J, Giaume C (2000) Activity-dependent neuronal control of GAP-junctional communication in astrocytes. J Cell Biol 149:1513-1526

Salhia B, Angelov L, Roncari L, Wu X, Shannon P, Guha A (2000) 
Expression of vascular endothelial growth factor by reactive astrocytes and associated neoangiogenesis. Brain Res 883:87-97.

Schipke CG, Ohlemeyer C, Matyash M, Nolte C, Kettenmann H, Kirchhoff F (2001) Astrocytes of the mouse neocortex express functional $N$-methyl-D-aspartate receptors. FASEB J 15:1270-1272.

Schipke CG, Boucsein C, Ohlemeyer C, Kirchhoff F, Kettenmann H (2002) Astrocyte $\mathrm{Ca}^{2+}$ waves trigger responses in microglial cells in brain slices. FASEB J 16:255-257.

Schwartz T, Rabinowtz D, Unni V, Kumar VS, Smetters DK, Tsiola A, Yuste R (1998) Networks of coactive neurons in developing layer 1. Neuron 20:541-552.

Spitzer NC, Lautermilch NJ, Smith RD, Gomez TM (2000) Coding of neuronal differentiation by calcium transients. BioEssays 22:811-817.

Stellwagen D, Shatz CJ (2002) An instructive role for retinal waves in the development of retinogeniculate connectivity. Neuron 33:357-367.

Stoop R, Pralong E (2000) Functional connections and epileptic spread between hippocampus, entorhinal cortex and amygdala in a modified horizontal slice preparation of the rat brain. Eur $\mathrm{J}$ Neurosci 12:3651-3663.

Tashiro A, Goldberg J, Yuste R (2002) Calcium oscillations in neocortical astrocytes under epileptiform conditions. J Neurobiol 50:45-55.

Thastrup O, Cullen PJ, Drobak BK, Hanley MR, Dawson AP (1990) Thapsigargin, a tumor promoter, discharges intracellular $\mathrm{Ca}^{2+}$ stores by specific inhibition of the endoplasmic reticulum $\mathrm{Ca}^{2+}$-ATPase. Proc Natl Acad Sci USA 87:2466-2470.

Venance L, Stella N, Glowinski J, Giaume C (1997) Mechanisms involved in initiation and propagation of receptor-induced intercellular calcium signaling in cultured rat astrocytes. J Neurosci 17:1981-1992.
Venance L, Prémont J, Glowinski J, Giaume C (1998) Gap junctional communication and pharmacological heterogeneity in astrocytes cultured from the rat striatum. J Physiol (Lond) 510.2:429-440.

Ventura R, Harris KM (1999) Three-dimensional relationships between hippocampal synapses and astrocytes. J Neurosci 19:6897-6906.

Verkhratsky A, Orkand RK, Kettenmann H (1998) Glial calcium: homeostasis and signaling function. Physiol Rev 78:99-141.

Vesce S, Bezzi P, Volterra A (1999) The active role of astrocytes in synaptic transmission. Cell Mol Life Sci 56:991-1000.

Willmott NJ, Wong K, Strong AJ (2000) A fundamental role for the nitric oxide-G-kinase signaling pathway in mediating intercellular $\mathrm{Ca}^{2+}$ waves in glia. J Neurosci 20:1767-1779.

Wong ROL (1999) Retinal waves and visual system development. Annu Rev Neurosci 22:29-47.

Yuste R, Katz LC (1991) Control of postsynaptic $\mathrm{Ca}^{2+}$ influx in developing neocortex by excitatory and inhibitory neurotransmitters. Neuron 6:333-344.

Yuste R, Peinado A, Katz LC (1992) Neuronal domains in developing neocortex. Science 257:665-669.

Zhuo L, Sun B, Zhang C-L, Fine A, Chiu S-Y, Messing A (1997) Live astrocytes visualized by green fluorescent protein in transgenic mice. Dev Biol 187:36-42.

Zhuo Q, Petersen CC, Nicoll RA (2000) Effects of reduced vesicular filling on synaptic transmission in rat hippocampal neurones. J Physiol (Lond) 525 1:195-206.

Zorzato F, Scutari E, Tegazzin V, Clementi E, Treves S (1993) Chlorocresol: an activator of ryanodine receptor-mediated $\mathrm{Ca}^{2+}$ release. Mol Pharmacol 44:1192-1201. 